\title{
How to model the neurocognitive dynamics of decision making: $A$ methodological primer with ACT-R
}

\author{
Cvetomir Dimov ${ }^{1,2}$ (D) $\cdot$ Patrick H. Khader ${ }^{3}$. Julian N. Marewski ${ }^{2} \cdot$ Thorsten Pachur $^{4}$
}

Published online: 8 August 2019

(C) The Psychonomic Society, Inc. 2019

\begin{abstract}
Higher cognitive functions are the product of a dynamic interplay of perceptual, mnemonic, and other cognitive processes. Modeling the interplay of these processes and generating predictions about both behavioral and neural data can be achieved with cognitive architectures. However, such architectures are still used relatively rarely, likely because working with them comes with high entry-level barriers. To lower these barriers, we provide a methodological primer for modeling higher cognitive functions and their constituent cognitive subprocesses with arguably the most developed cognitive architecture today-ACT-R. We showcase a principled method of generating individual response time predictions, and demonstrate how neural data can be used to refine ACT-R models. To illustrate our approach, we develop a fully specified neurocognitive model of a prominent strategy for memory-based decisions - the take-the-best heuristic - modeling decision making as a dynamic interplay of perceptual, motor, and memory processes. This implementation allows us to predict the dynamics of behavior and the temporal and spatial patterns of brain activity. Moreover, we show that comparing the predictions for brain activity to empirical BOLD data allows us to differentiate competing ACT-R implementations of take the best.
\end{abstract}

Keywords ACT-R $\cdot$ fMRI $\cdot$ Response times $\cdot$ Model comparison

If a theory covers only one part or component, it flirts with trouble from the start.

- A. Newell (1990, p. 17)

Alphabetical author order. Patrick H. Khader, Julian N. Marewski, and Thorsten Pachur contributed equally to this work

Electronic supplementary material The online version of this article (https://doi.org/10.3758/s13428-019-01286-2) contains supplementary material, which is available to authorized users.

Cvetomir Dimov

cdimov@andrew.cmu.edu

1 Department of Psychology, Carnegie Mellon University, Pittsburgh, PA, USA

2 Department of Organizational Behavior, University of Lausanne, Lausanne, Switzerland

3 Department of Psychology, Brandenburg Medical School, Neuruppin, Germany

4 Max Planck Institute for Human Development, Berlin, Germany
Research in cognitive psychology aims at identifying the mental processes that produce observable behavior - in J. R. Anderson's (1990) terms, to find "the function that maps input to output" (p. 24). Yet perceptual, mnemonic, and other cognitive processes typically generate behavior in interplay with higher cognitive functions. A prime example of such higher cognitive functions are processes of decision making. When trying to understand the processes underlying decision making, cognitive scientists have been relying not only on data about the decision outcome, but also on various types of data potentially indicative of the decision process itself. Such process data include response times (RTs), measures of predecisional information search (e.g., eye tracking, Mouselab; Duchowski, 2002; Willemsen \& Johnson, 2011), or verbal protocols (Ericsson \& Simon, 1980). From those data, researchers try to infer what kind of decision mechanisms drove a persons' choices. Yet, in doing so, they face a conundrum: Due to the abovementioned interplay of cognitive subprocesses, the observed RTs and eye movements are not only a function of decisional, but also of perceptual, attention$\mathrm{al}$, and mnemonic processes.

In addressing this conundrum many researchers try to keep all nondecisional processes constant in an experiment and rely on supplementary assumptions about these processes to 
evaluate the extent to which observations are consistent with a given decision mechanism. Those assumptions come in different forms. For example, some are explicit assumptions about memory, such as that the time to judge an object as recognized will vary as a function of frequency of occurrence in the media (e.g., Hertwig, Herzog, Schooler, \& Reimer, 2008). Others are assumptions about reading phases and keystroke times, which are often assumed to be constant and then subtracted from observed RTs (e.g., Johnson, SchulteMecklenbeck, \& Willemsen, 2008; Pachur, Hertwig, Gigerenzer, \& Brandstätter, 2013). Moreover, assumptions are embedded within data analysis procedures, such as that the probabilities of committing an error are equal for all decision trials (trembling-hand error; e.g., Bröder, 2003) or that RTs are log-normally distributed (e.g., Glöckner, 2009). Finally, approaches differ in terms of their specificity and complexity, ranging from informal (i.e., verbal) assumptions about the average duration of potential arithmetic and reading operations (Payne, Bettman, \& Johnson, 1993) to formal (i.e., mathematical or computational) theories of, for example, memory (e.g., Dougherty, Gettys, \& Ogden, 1999).

Although such approaches have been followed to identify specific regularities in the observed data speaking to different cognitive processes of interest, very few researchers actually model how those cognitive processes dynamically interplay and, consequently, produce the observable RTs, eye movements, and other process data. Yet this lack of models can be problematic when attempting to identify specific cognitive mechanisms. Is a model's description of the data (in)adequate because of the main or because of the supplementary assumptions (see the epigram above)? What has been called the irrelevant-specification problem (Lewandowsky, 1993; A. Newell, 1990) ${ }^{1}$ revolves around the dilemma of whether assumptions make psychological claims or whether they merely serve to enable the generation of predictions. In the worst case, incorrect assumptions will lead to patterns in the observed data being wrongly attributed to the cognitive process of interest (e.g., decisional processes).

Moreover, although behavioral data-including outcome data (i.e., overt decisions) and nonphysiological process data (i.e., RTs, eye movements, verbal protocols) —inform us about at least some aspects of a cognitive task, they do not provide sufficient constraints to unequivocally identify the underlying cognitive subprocesses, because "there is an infinite number of mechanisms that compute the same input-output function" (J. R. Anderson, 1990, p. 24). In fact, Anderson's critique, known as the identifiability problem, puts into question any process model that is developed and tested only through

\footnotetext{
${ }^{1}$ That is, when translating an underspecified theory into a precise formal model, many details have to be specified in the code that are not part of the original theory. The irrelevant-specification problem poses the question of which of those details can be considered part of a psychological theory, and which should not be.
}

behavioral data. Instead, he contends, we need the type of neural data that "trace[] out the states of computation in the brain" (J. R. Anderson, 1990, p. 25) to pin down the mental steps a participant goes through. Ideally, taking into account neural data would allow researchers to deduce the dynamics of these cognitive processes from physiological data on the temporal and spatial patterns of brain activity. However, how can task-related activity in specific brain regions be related to dynamically interplaying cognitive mechanisms?

\section{Cognitive architectures}

A formal description of the temporal and spatial patterns of brain activity in different tasks can be provided by cognitive architectures. A cognitive architecture is a quantitative model that applies to many different behaviors and that casts theories of memory, perception, action selection, and other components of cognition into a single mathematical or computational system (for an introduction to cognitive architectures, see, e.g., Gluck, 2010). At present, the most detailed cognitive architecture is $A C T-R$ (J. R. Anderson, 2007; for other architectures, see, e.g., Eliasmith, 2013; Meyer \& Kieras, 1997; A. Newell, 1990). ACT-R has been continuously developed and updated over the last decades to incorporate current findings and theoretical ideas in a principled manner. ACT-R simultaneously generates multiple types of quantitative behavioral and neural data, ranging from RTs and eye movements to functional magnetic resonance imaging (fMRI) or electroencephalography (EEG) data. Indeed, ACT-R is being increasingly used in combination with neural data to link neural activation patterns with specific cognitive processes (see J. R. Anderson, Fincham, Qin, \& Stocco, 2008). Moreover, neural data have been relied on to more rigorously test cognitive models composed of those cognitive processes (see Borst \& Anderson, 2015, for an overview of these approaches). All this makes ACT-R an excellent tool to address the irrelevant specification and identifiability problems.

ACT-R is widely used by a large worldwide community and applied to areas as diverse as airplane flying (Byrne \& Kirlik, 2005), intelligent tutoring (Ritter, Anderson, Koedinger, \& Corbett, 2007), skill acquisition (Taatgen, Huss, Dickison, \& Anderson, 2008), and list memory (J. R. Anderson, Bothell, Lebiere, \& Matessa, 1998; see http://act-r. psy.cmu.edu/publication/ for a complete list of publications). Yet, building a model in ACT-R comes with at least three important entry-level barriers. First, users need to fully understand the theory. This is not trivial, because ACT-R consists of models of various aspects of cognition (e.g., memory, perception, procedural knowledge) and their interaction. Second, users need to know how to implement their hypotheses in this computational-modeling framework, which is instantiated as a programming language with built-in human constraints, 
written in Common Lisp. Third, users need to be aware of methods for developing, calibrating, and testing complex architectural models.

Our goal in this article is to guide scientists in modeling process data with ACT-R. In offering this methodological primer, we complement the extensive tutorial that comes with the ACT-R software (available at http://act-r.psy.cmu.edu/ software/) and the excellent step-by-step tutorial of how to use the architecture with fMRI data (Borst \& Anderson, 2017) in three ways. First, we illustrate how ACT-R model parameters can be empirically constrained on separate experimental tasks in a principled manner. Second, we showcase the capability of these constrained models to generate participant-specific behavioral predictions (e.g., RT distributions). Third, we illustrate how BOLD data can help to further refine a model beyond what can be achieved from behavioral data alone.

We will first introduce the formal underpinnings of ACT-R and demonstrate how to develop a neurocognitive model for a prominent strategy in memory-based decision making, the take-the-best (TTB) heuristic (Gigerenzer \& Goldstein, 1996). To develop our model, we will rely on an fMRI experiment in which participants were instructed to follow that decision strategy (Khader et al., 2011, Exp. 1). We will demonstrate how the parameters of our model can be empirically estimated via a behavioral task in that study that precedes the actual decision task. We will then generate individual predictions about RTs in the decision task and compare those to the empirical data. Finally, we will showcase how to use the BOLD predictions of our model to further refine it.

\section{Overview of ACT-R}

To model and predict behavior and brain activation with ACT$\mathrm{R}$, different cognitive processes are modeled by separate modules, which have been mapped onto different brain areas (see Fig. 1). These modules include perceptual ones - namely, a visual and an aural module, which model focused attention to perceptual input and are mapped to regions reflecting advanced perceptual processing: the secondary auditory cortex and fusiform gyrus, respectively. There are also vocal and manual modules, which model speech and typing on a keyboard, respectively. These output modules are mapped onto two regions in the central sulcus, where the face and tongue and the hand are represented. Furthermore, there are three central cognitive modules: The goal module tracks an agent's goals; this module maps onto the anterior cingulate cortex. The imaginal module holds information relevant to the task and problem state at hand. This module corresponds to posterior parietal cortex - a region hypothesized to be involved in the transformation of mental representations. How information is stored in and retrieved from declarative memory is modeled by the declarative module. This module is associated with the lateral inferior prefrontal cortex. All modules can operate in parallel, but within each module, information is processed serially (Byrne \& Anderson, 2001).

The perceptual and central cognitive modules operate on declarative knowledge (i.e., explicit memory). Such knowledge is modeled by chunks. Chunks represent information input from the visual and aural modules, the current goal of the cognitive system, information relevant to the problem state as well as knowledge in long-term memory. Chunks are collections of attributes, called slots, and their corresponding values:

(chunk-name ${ }^{2}$ slot1 slot1-value slot2 slot2-value slot3 slot3-value ....).

For example, we can represent factual knowledge, such as "Paris is the capital of France," or current states of the world, such as "the bird sings loudly," with the following two chunks:

(capital-France name Paris role capital country France) (loud-bird-by-my-office object bird action sings adverb loudly).

A procedural module orchestrates the other modules and functions as the central bottleneck in information processing (Fig. 1). The procedural module is associated with the basal ganglia - a system hypothesized to implement conditional information routing to the cortex (Stocco, Lebiere, \& Anderson, 2010). This module is instantiated as a production system (i.e., A. Newell, 1973a); that is, it consists of a collection of production rules (if-then rules). The productions' conditions (the "if" parts of the rules) are matched against the current state of the other modules (e.g., whether something is retrieved from memory or whether an object is visually attended to). Production rules, whose conditions are met, can fire; that is, they can direct other modules to change their current state. Examples of production rules in natural language are:

1. IF an object is visually attended to and the goal is to look at it,

THEN visually encode the object of attention.

2. IF the goal is to guess the name of a country's capital and France is currently stored into the problem state,

THEN attempt to recall the name of France's capital.

Production rules do not access modules' contents directly, but via buffers. Buffers serve as communication channels

\footnotetext{
${ }^{2}$ For convenience, chunk names (e.g., capital-France) are used in ACT-R to refer to chunks. Those names are not considered to form part of the chunk itself.
} 


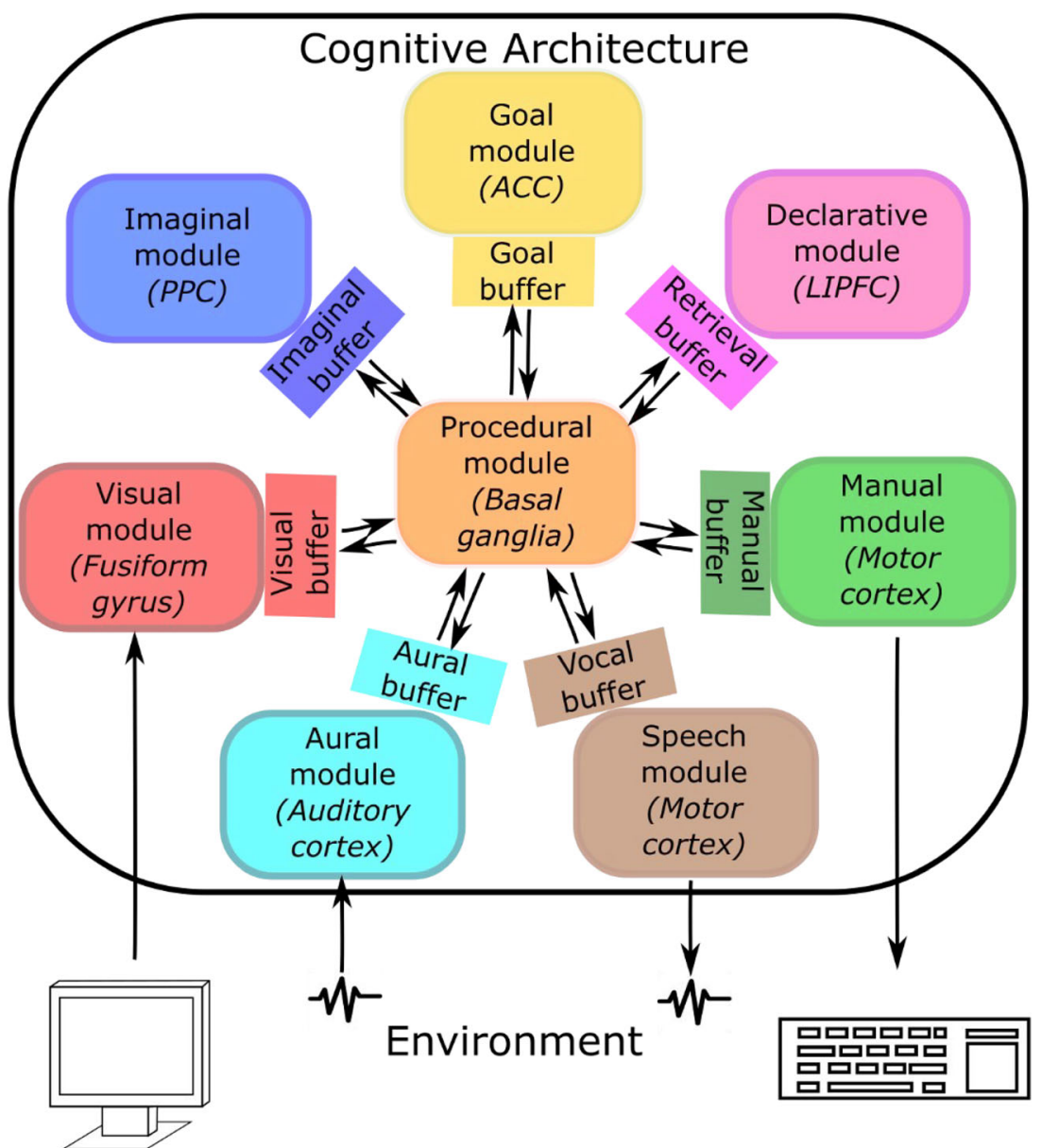

Fig. 1 The modular organization of the cognitive system and module-tobrain mappings according to the ACT-R cognitive architecture. Seven modules interact with each other through an eighth, procedural module. The procedural module communicates with the other modules through buffers, represented as small rectangles. The cognitive architecture

between modules and productions, and as such can create a bottleneck for information transfer (Salvucci \& Taatgen, 2008). For example, if the procedural module needs to access information in the visual field, the visual module has to first place that information (in the form of a chunk) into the visual buffer. Likewise, when a production rule sends a retrieval request to the declarative module, the retrieved information (i.e., a chunk) must first be placed in the retrieval buffer before another production rule can utilize it. In essence, when an ACT-R model is run, requests are sent to modules, which leads the modules to execute operations. After completing those operations, modules can place chunks into their respective buffers. Reversely, production rules await those chunks to be placed into specific buffers to match the conditions specified in their "if" part. Once those conditions are met, the rules can fire (i.e., execute their "then" part) and send further processing requests to modules. The serial operation of modules and the time that it takes them to complete their operations creates delays and bottlenecks in the system. Altogether, this interacts with the environment (e.g., in an experiment: with a computer screen and/or a keyboard) through its perceptual and motor modules. $\mathrm{ACC}=$ anterior cingulate cortex LIPFC $=$ lateral inferior prefrontal cortex; $\mathrm{PPC}=$ posterior parietal cortex

complex interaction between the procedural and other modules (Fig. 1) produces behavior.

\section{ACT-R's subsymbolic system}

The modules and buffers can be best thought of as an "upper" symbolic layer of the architecture. ACT-R distinguishes that symbolic system from a "lower" layer, called the subsymbolic system. The subsymbolic system shapes the outcome of each module's and each buffer's operations. Specifically, the subsymbolic system describes memory retrieval, the selection of different production rules, visual and other processes in terms of a series of mathematical equations. Those equations determine, for example, how likely and how quickly memories can be retrieved, which of several alternative courses of action will be executed, or how long a keypress will take.

An important component of ACT-R's subsymbolic system are the equations governing the retrieval of memory traces (i.e., of chunks). Altogether, these equations cast memory as 
an information-processing device that systematically exploits the statistical patterns of occurrence of stimuli in the world. Specifically, memory can be thought of as inferring the probability that a memory trace of stimuli will be needed, on the basis of the history of past encounters with those stimuli, in order to achieve future processing goals. The history of past encounters, in turn, probabilistically hinges on patterns of occurrence of those stimuli in the world. For instance, we are more likely to learn about car brands and cities that occur more often in the media. In real-world environments, patterns of the past occurrence of stimuli are predictive of future ones (e.g., J. R. Anderson \& Schooler, 1991; Schooler \& Anderson, 1997). To illustrate this, the more often an object (e.g., a name) has been mentioned in the news in the past, the more likely it is that this object will be mentioned again in the future. Similarly, the longer it has been since an object has last been mentioned, the less likely it is that the object will be mentioned again in the future. These lawful relations in information occurrence in the environment allow memory to guide current information-processing demands - for example, by retrieving memory traces of recently encountered stimuli more quickly, or by setting aside (i.e., forgetting) information that has been encountered infrequently or a long time ago.

Specifically, in ACT-R each chunk $i$ has an activation, $A_{i}$, associated with it that quantifies the strength of that memory trace. Activation models the likelihood that a chunk is needed to achieve a given processing goal at the current moment. Activation itself is fed by three subcomponents - the chunk's base-level activation, $B_{i}$, the spreading activation, $S A_{i}$, and noise, $\varepsilon$ :

$A_{i}=B_{i}+S A_{i}+\varepsilon$

The base-level activation is a function of the chunk's history:

$B_{i}=\ln \sum_{k=1}^{n} t_{k}^{-d}$

where the decay parameter, $d$, specifies the rate forgetting over time, which is modeled in terms of a power function. The parameter $n$ represents the number of encounters with the information that chunk $i$ represents, and $t_{k}$ is the time since the $k$ th encounter. The latter two parameters capture the history of encountering stimuli in the world.

$S A_{i}$ quantifies a chunk's relevance in the current context by assuming that chunks related to what is currently the focus of attention are more likely to be needed than those that are not. In ACT-R, context is modeled as all chunks currently stored in the buffers. Thus, spreading activation to chunk $i$ in declarative memory is a function of the associations between that chunk and the chunks $j$ currently in the buffers:

$S A_{i}=\sum_{j} W_{j} S_{j i}$
The amount of spreading activation $S A_{i}$ is determined by the associative strength, $S_{j i}$, between chunks $i$ and $j$, which is weighted by the source activation, $W_{j}$, of chunk $j$ in a buffer. The associative strengths, $S_{j i}$, between chunks is approximated by

$S_{j i}=S-\ln \left(\operatorname{fan}_{j}\right)$,

where $S$ denotes the maximum associative strength and $\mathrm{fan}_{j}$ is the number of other chunks associated with a chunk $j$. The more chunks are associated with a chunk in memory, the lower the associative strength between it and each of its associates becomes.

Finally, retrieval noise, $\varepsilon$, is added to the base-level and spreading activation components when a retrieval request is made. The value of $\varepsilon$ is generated from a logistic distribution with a mean of zero and a variance of

$\sigma_{d}^{2}=\frac{\pi^{2}}{3} s_{d}^{2}$

where $s_{d}$ is a free parameter. The random nature of activation means that at each specific time a chunk can have an activation higher or lower than its mean.

A chunk can be retrieved only when its current activation value is above the retrieval threshold, $\tau$. The retrieval probability, $p_{i}$, of a chunk is the probability that its activation exceeds this threshold:

$p_{i}=\frac{1}{1+e^{-\frac{\mu_{A_{i}}-\tau}{s}}}$,

where $\mu_{A_{i}}=B_{i}+S A_{i}$ is the mean of the activation distribution. The time required for retrieval is scaled by a latency factor $F$ :

$t_{\text {retrieval }}=F e^{-A_{i}}$.

Thus, more active chunks are more quickly retrieved. If no chunk matches a retrieval request or if the matching chunk with the highest activation is below the retrieval threshold, a retrieval failure will occur. The retrieval failure time is

$t_{\text {retrieval failure }}=F e^{-\tau}$.

Another important component of the subsymbolic system is the equations governing ACT-R's production rules. Various higher-order cognitive functions are implemented in ACT-R as sets of productions that specify, for instance, what the goals of the decision maker are (e.g., making inferences about car brands as accurately as possible), when and how that person will encode information in his/her environment (e.g., reading the names of different car brands in a catalogue or on a computer screen) and when and how the participant will search for information in memory (e.g., what facts about different cars 
will be recalled). These equations determine which production rules will be executed in case the conditions (i.e., the "if" parts) of several of those if-then rules are met. According to these equations, the productions that have been most successful in the past are the ones that are most likely to be chosen, with production success being quantified by its utility. A production's utility is learned according to the Rescorla-Wagner learning rule (Rescorla \& Wagner, 1972):

$U_{i}(n)=U_{i}(n-1)+\alpha\left[R_{i}(n)-U_{i}(n-1)\right]$,

where $U_{i}(n)$ is the utility of the production after its $n$th application, $U_{i}(n-1)$ is its utility after the $(n-1)$ th application, and $R_{i}(n)$ is the reward that it receives on the $n$th application. Basically, upon each application of a production, its utility is updated in the direction of the reward that it receives: If the reward is lower than its current utility, the utility will decrease, whereas if it is higher, it will increase.

\section{ACT-R and brain activation}

To derive predictions regarding brain activation, ACT-R draws on the relationship between brain activity and blood supply (e.g., Boynton, Engel, Glover, \& Heeger, 1996): Metabolic demand in an active brain region leads to an increased blood supply to that region, measured as a hemodynamic response (HR). The HR is not immediate, but peaks around $6 \mathrm{~s}$ after the metabolic demand. Its temporal profile, labeled the hemodynamic response function $(H R F)$, is described by a gamma distribution or a mix of two gamma distributions. Here, we will use the canonical $H R F$ as implemented in the SPM fMRI analysis software ("statistical parametric mapping"; Friston et al., 1998; see Fig. 2 for a visualization of this $H R F$ ):

$H R F_{\mathrm{SPM}}(t)=\frac{6 t^{5} e^{-t}}{\Gamma(6)}-\frac{1}{6} \frac{16 t^{15} e^{-t}}{\Gamma(16)}$,

where $\Gamma$ is the gamma function.

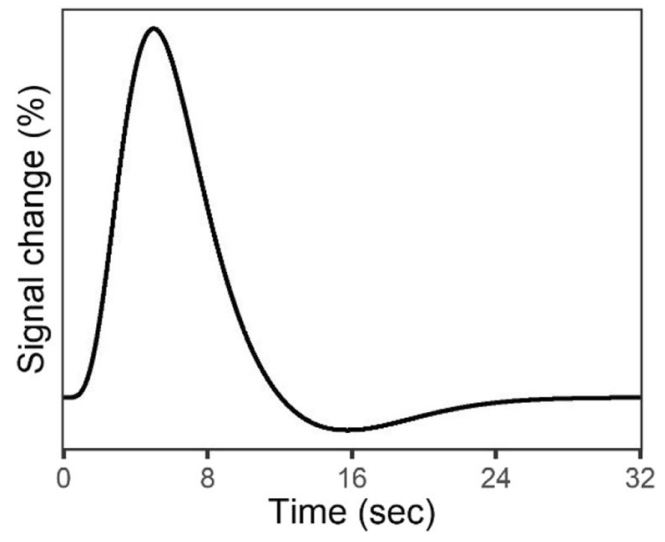

Fig. 2 SPM's canonical hemodynamic response function
Once an ACT-R model has been developed, generating BOLD response predictions involves the following steps: First, the activity of each module is described with a demand function $D(t)$. Here, we follow the standard assumption that whenever a module is active $[D(t)=1]$, the brain region associated with this module is active [else, $D(t)=0$ and the brain region is inactive]. We then assume that at each moment when that brain region is active, it responds according to the $H R F$ in Eq. 10 (see Borst \& Anderson, 2017). The resulting HR prediction is a convolution of the demand function over the entire experiment, and that $H R F$ :

$H R(t)=(D * H R F)(t)$.

The HR predictions can be related to brain activation data in two ways: model-based fMRI analysis and region-ofinterest (ROI) analysis (e.g., Borst \& Anderson, 2015). Recall that ACT-R's modules are assumed to mediate different cognitive functions (e.g., declarative memory, vision). In model-based fMRI analysis, the blood-oxygenation-leveldependent (BOLD) response prediction of each ACT-R module is regressed against all voxels in the experimental data, which, in turn, allows identifying the brain correlates of the modules. This approach has been used to identify regions in the brain that strongly correlate with module activity.

Whereas model-based fMRI analysis serves to establish module-to-brain mappings, ROI analysis uses alreadyestablished mappings to evaluate cognitive models. Specifically, ROI analysis compares BOLD predictions associated with different cognitive processes to brain activation in predetermined regions. ${ }^{3}$ In contrast to analyses involving behavioral data, an ROI analysis of neural data is especially useful for testing cognitive models (e.g., J. R. Anderson et al., 2008; Borst et al., 2010), because neural data relate directly to each individual module's activity.

Next, after introducing the decision strategy that will serve as a case-in-point-TTB - we will demonstrate how ACT-R models work and how predictions for that strategy can be derived and tested. We will use both behavioral data and neural data in a ROI analysis to test the detailed cognitive model of TTB.

\section{An example of decision processes: The TTB heuristic}

TTB is a representative of an important class of decision strategies that implement sequential information search. TTB and

\footnotetext{
${ }^{3}$ For the Talairach coordinates of those brain regions, see J. R. Anderson, Fincham, et al. (2008). For the Montreal Neurological Institute coordinates, see Borst, Taatgen, Stocco, and van Rijn (2010). Note that Borst, Nijboer, Taatgen, van Rijn, and Anderson (2015) have further refined those brain mappings through a data-driven, model-based approach.
} 
similar lexicographic models, such as elimination by aspects (Tversky, 1972), stand in contrast to the classic assumption that people integrate and weight the available evidence to make decisions (Chase, Hertwig, \& Gigerenzer, 1998), such as in subjective expected utility theory (Edwards, 1954) or other compensatory weighted-additive strategies (e.g., Payne et al., 1993). TTB is a model of inference: It uses objects' (e.g., cell phones, car brands, cities) attributes (e.g., whether a cell phone is recommended by others) to infer which of two objects has the larger value on an unknown criterion (e.g., the phone's quality). To this end, TTB operates on attributes with binary attribute values that are coded as 1 if positive (e.g., phone is recommended) or 0 if unknown or negative (not recommended). When making inferences, TTB inspects attributes in order of their importance. Once two objects have different values on an attribute $i$ (i.e., one has a value of 1 , the other of 0); that is, once a discriminating attribute is found, TTB makes a decision without considering further information (i.e., other attributes). In the literature, this decision process has been described in terms of three building blocks (e.g., Gigerenzer \& Gaissmaier, 2011):

Search rule: Search through attributes in the order of their validity.

Stopping rule: Stop search as soon as an attribute is found that discriminates between the objects.

Decision rule: Infer that the object with the positive attribute value has the higher value on the criterion of interest.

TTB has been shown to be used spontaneously in particular under conditions of high information cost, including memory-based (rather than screen-based) decisions (e.g., Bröder \& Gaissmaier, 2007; Bröder \& Schiffer, 2003). The notion underlying TTB, that people sometimes ignore information, has triggered a large number of empirical studies (Bergert \& Nosofsky, 2007; Bobadilla-Suarez \& Love, 2018; Bröder \& Schiffer, 2003; Juslin, Jones, Olsson, \& Winman, 2003; Khader, Pachur, \& Jost, 2013; Pachur \& Aebi-Forrer, 2013; Pachur \& Marinello, 2013; Rieskamp \& Otto, 2006; for an overview, see Pachur \& Bröder, 2013). To test TTB against information integration models, such as compensatory weighted-additive strategies, those studies have made use of RTs (Bröder \& Gaissmaier, 2007) and patterns of information search (B. R. Newell, Weston, \& Shanks, 2003), often making the kind of supplementary assumptions about mnemonic, perceptual, and motor processes that we mentioned in the introduction. Indeed, as can be seen, the search, stopping, and decision rules used in the literature to describe TTB remain fully silent about how the decisional processes assumed by TTB nestle into the rest of the cognitive architecture. Thus, developing an ACT-R implementation of TTB illustrates how to integrate TTB's key theoretical assumptions about decision making (such as sequential and limited search) with mnemonic, visual, and other information-processing activities.

When developing an ACT-R model of TTB - or any other decision mechanism, for that matter - to unveil the cognitive processes behind people's decisions, it is important to ensure that the observed output on every instance (e.g., a trial in an experiment) is produced by that mechanism and not by another one. In the cognitive and decision sciences, many experiments present participants with a decision task and then, on the basis of their responses, individual participants are classified, for instance, as "users" of TTB or alternative decision mechanisms (Bröder, 2000; B. R. Newell \& Lee, 2011; Nosofsky \& Bergert, 2007). Although suited for testing competing models of decision making against each other, such data make it difficult to develop architectural models of TTB, because the observed data can, but need not be produced by TTB. A data set in which participants' reliance on TTB is ensured has been provided by Khader et al. (2011). In their experiments, participants were instructed to rely on TTB for their decisions while their brain activity was recorded, which renders this data set an excellent basis for illustrating how neurocognitive ACT-R models can be developed.

\section{Developing an ACT-R implementation of TTB}

Khader et al. (2011) employed a memory-based paradigm in which two objects (here, a pair of fictitious companies) are presented on a computer screen and participants have to rely on their memory to recall previously learned attribute values and make a decision according to TTB. An ACT-R implementation of TTB should perform the same operations that a participant would: For example, it needs to read the object names and then recall objects' attribute values in order to make a decision.

The attribute values of objects are stored as chunks in the model's declarative memory. What slots would these chunks consist of? In Khader et al.'s (2011) experiment, prior to the decision task participants learned to associate objects (i.e., companies) with attributes (e.g., where the company is located and which product it produces) and their values (i.e., whether the attributes are positively or negatively related to the decision criterion; see Khader et al., 2011, for details). In our model, we rely on the following chunk structure to describe object attributes stored in memory:

(objectN-attributeM object-name objectN attributename attributeM attribute-value 0/1).

How are such chunks used to develop a model of TTB? In modeling participants' declarative memory, in a first step, the model's declarative memory is populated with all attribute 
values that participants learned prior to working on the decision task.

Once we have defined the declarative chunk structure, we continue with outlining the sequence of steps that a model needs to go through. In addition to TTB's search, stopping, and decision rules (see the section above on the TTB heuristic), we need to include all steps that a participant in an experiment would go through, such as visual and motor steps. For our task, these steps are (1) look at the company names, (2) retrieve the attribute, (3) retrieve the corresponding attribute values, (4a) press the key on the keyboard that corresponds to the company with a positive attribute value if the attribute values differ, or (4b) retrieve the next attribute in the hierarchy if the attribute values on the current attribute are the same.

To translate these steps into a sequence of productions, it is necessary to consider ACT-R's architectural constraints. For example, the visual module can only process objects serially. This means that the visual system needs to attend the first company name on the screen-an action guided by a production - and then encode that company name guided by a second production. Only then can it attend and encode the second company name. Similar buffer capacity and temporal constraints exist for other modules. For instance, the imaginal module can also only perform one operation at a time (e.g., store a chunk or modify the chunk it currently holds) and that operation also incurs a time cost (i.e., $200 \mathrm{~ms}$ ). Similarly, the retrieval module can only be attempting to retrieve one chunk from long-term memory, and the time it takes to perform the retrieval is determined by Eq. 7 .

Figure 3 shows a process trace of a run of the entire ACT-R implementation of TTB for a decision on which the most valid attribute in the hierarchy discriminates between the two companies. ${ }^{4}$ The components of the cognitive architecture are active at different points in time, with eight production rules coordinating the modules' actions. In this example, the model starts by comparing the two objects on the screen: It first looks at the left part of the screen (guided by Production 1), reads the name of the company present on that part of the screen (Production 2 ), and stores it in the imaginal buffer while shifting its gaze to the right part of the screen (Production 3). After reading (Production 4) and storing (Production 5) the name of the company present there, the model checks whether the companies are different, and if so, it executes TTB, starting with the most valid attribute (Production 6). For the most important attribute, the model recalls the attribute value of the left company (also Production 6), then it recalls the attribute value of the right company (Production 7), and finally it compares them (Production 8). Then the model chooses the company to which that attribute is pointing, and finishes with an overall RT of $2 \mathrm{~s}$. Note that on other trials, retrieval might be faster or slower, or

\footnotetext{
${ }^{4}$ See the model "TTB_v1.lisp," in the online materials at osf.io/25pt8, for the model code.
}

more attributes might need to be examined prior to making a decision, which will lead to a different $\mathrm{RT}$ and a different relative activity of each module.

\section{Testing the ACT-R model of TTB}

In an initial learning task, the 17 participants memorized the values on four attributes about 16 fictitious companies; a total of 64 attribute values. In a subsequent strategy-training task, participants were instructed how to use TTB. They also practiced to apply this heuristic, using a decision task different from that in the main decision task (a fictitious job-scenario). Finally, participants learned the hierarchy of the four attributes by indicating their importance repeatedly. In the decision task, participants' responses and the associated RTs and BOLD signals were recorded. A total of 132 company pairs were presented (on the left and right sides of the screen, respectively) in three blocks of 44 trials each. Participants were instructed to use TTB and the acquired attribute knowledge to decide which of each two companies is more likely to be successful in the future. The intertrial interval (ITI) was 2, 4, or $6 \mathrm{~s}$ (varied randomly), and each trial started with a fixation cross presented for $2 \mathrm{~s}$.

There were five types of decision trials, which differed in the number of attributes that TTB would need to consider prior to making a decision (i.e., none, one, two, three, or all four attributes). This is relevant because it is assumed that, due to TTB's stopping rule, the time it takes to make decisions with this strategy depends on how many attributes have been considered before a discriminating attribute is retrieved from memory (e.g., Bröder \& Gaissmaier, 2007). In control trials (i.e., where no attributes need to be considered), the same company name was presented on both sides of the screen, and participants were instructed to respond directly, without retrieving any attributes. For further details on the experimental methodology, see Khader et al. (2011).

\section{A roadmap for model testing}

We used data from Khader et al.'s (2011) Exp. 1 to develop and test an ACT-R implementation of TTB. Figure 4 provides an overview of the various steps and stages of the procedure. ${ }^{5}$ First, we calibrated the model by fitting its free parameters in the learning task. To this end, we developed a recall model in ACT-R. We then used the estimated parameters from the learning task to generate distributional RT predictions for the decision task with the TTB implementation. Note that the parameters were not fitted to the data of the decision task, so these are genuine predictions. We also performed an ROI analysis to compare brain activation predictions to BOLD

\footnotetext{
${ }^{5}$ The data, analysis scripts, and model files are available at osf.io/25pt8.
} 

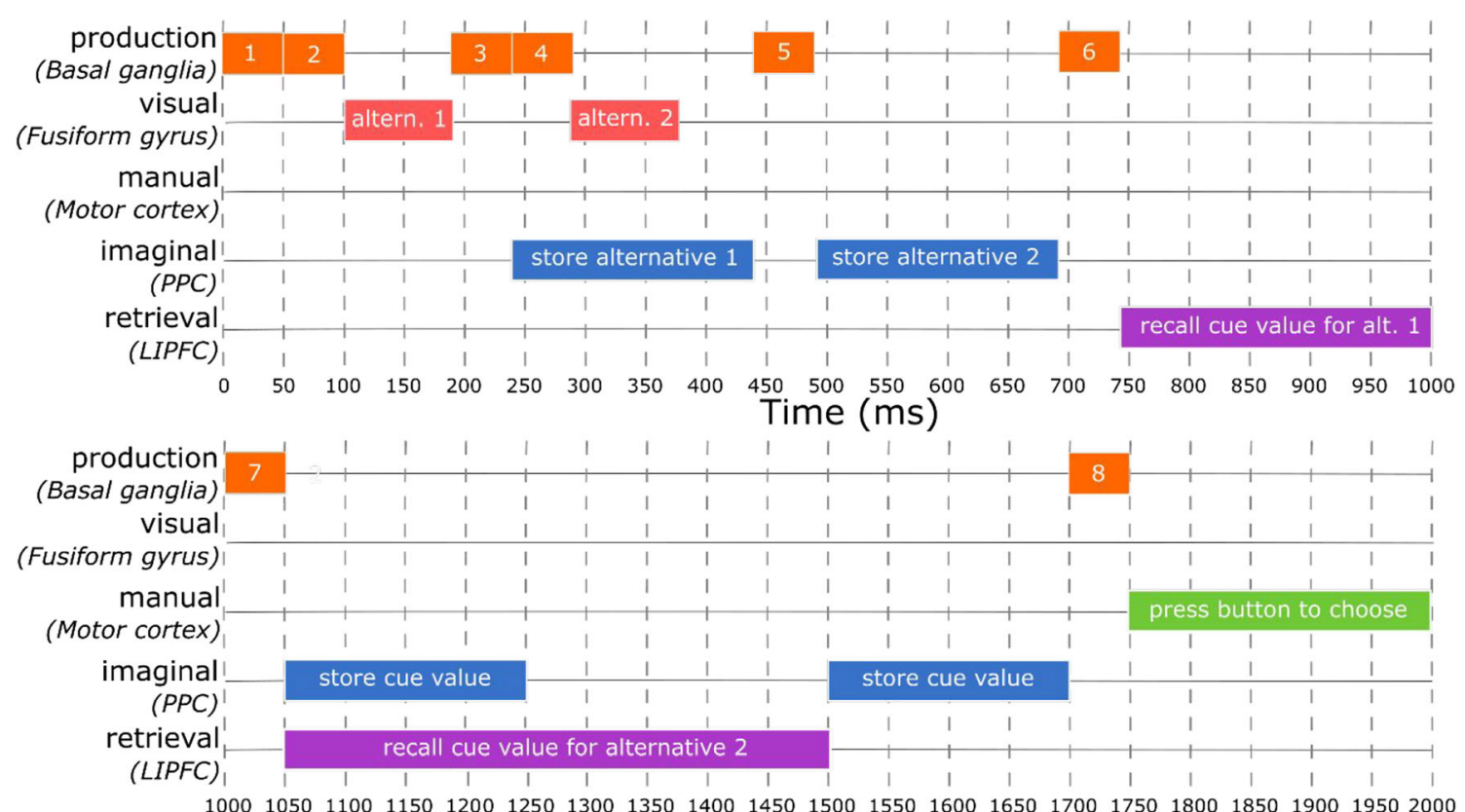

100010501100115012001250130013501400 Time (ms)

Fig. 3 Schematic process trace of an ACT-R implementation of TTB. For the sake of illustration, the model trace schematically depicts arbitrary recall times (for actual times, run the model in the online materials at osf.io/25pt8). The $y$-axis denotes various ACT-R modules and the associated brain regions. Eight production rules control the behavior of this model. Production 1 directs visual attention to the location of the first object (Company 1), and Production 2 requests that the visual module encode it. Productions 3 and 4 repeat the same steps for the second object

data. Finally, in an iterative process, we used the ROI analysis to further refine our model. Specifically, we constructed seven alternative implementations of TTB and selected the implementation whose processing steps generated BOLD predictions best corresponding to observed data.

\section{Model calibration in the learning task}

To rigorously test a model's descriptive power, it is important to ensure that it performs well in predicting data out-of-sample rather than being able to fit data (e.g., Pitt, Myung, \& Zhang, 2002). To derive predictions about RTs and BOLD responses in the decision task, we estimated ACT-R's memory parameters for each participant from his/her RTs in the learning task (Fig. 4, steps 1 and 2). During the learning task, different attribute values are remembered increasingly better across various rounds of learning (see the description of the learning task above), until the corresponding chunks are activated strongly enough to be retrieved with a probability of almost 1 (see Eq. 6) in the last round of learning. Thus, the last learning round defines the peak activation of each attribute value chunk. Moreover, it is temporally closest to the decision task. This renders a chunk's activation at this point in time a reasonable approximation to that chunk's activation at the beginning of the decision task.
(Company 2) and also request storing the first object in the imaginal buffer. Production 5 starts storing the name of the second object in the imaginal buffer. Productions 6 and 7 request that the declarative module retrieve the attribute values of the first attribute for the two objects. Finally, Production 8 selects the object with a positive attribute value and requests a keypress. LIPFC $=$ lateral inferior prefrontal cortex. PPC $=$ posterior parietal cortex

ACT-R relates activation to RT as per Eq. 7. We transformed this equation to estimate the activation of chunks representing attribute values from the retrieval time of each attribute value chunk in the last round of learning:

$A=-\log \frac{t_{\text {retrieval }}}{F}$,

where the latency factor, $F$, is left fixed at its default value ( $F=$ 1). This implies that estimating activation as per Eq. 12 requires no parameter fitting. ${ }^{6}$ To assess retrieval time, we then assumed that

$t_{\text {retrieval }}=\mathrm{RT}-t_{\text {non-retrieval }}$

that is, that the total RT consists of separable retrieval and nonretrieval components. We then estimated $t_{\text {nonretrieval }}$ by constructing an ACT-R model of recall in a learning trial, whereby we relied entirely on ACT-R's default parameters (Fig. 4, Step 1). This model, shown in Fig. 5, starts by looking at the company name. It then stores that name in the imaginal buffer and looks at the attribute. Once both

\footnotetext{
${ }^{6}$ ACT-R includes parameters that shape the workings of its subsymbolic system. For example, these parameters determine how long retrieval takes, or low quickly one would attend an object in the visual field. These parameters have default values, which ensure consistency between models constructed within this architecture.
} 

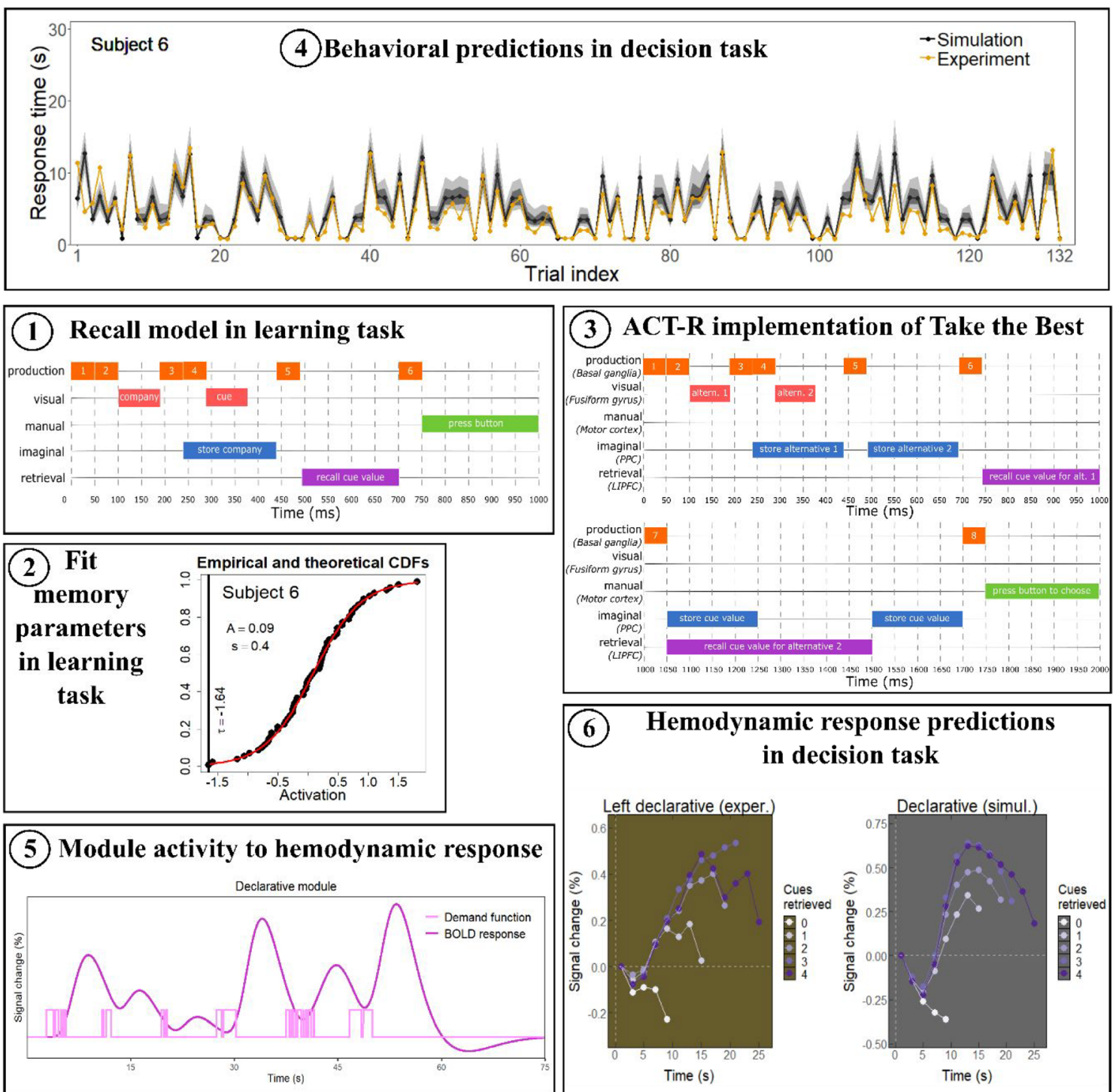

Fig. 4 Overview of the different steps of model development and testing. (1) An ACT-R model of recall is developed to estimate perceptual-motor times in the learning task. (2) Memory parameters are estimated from the learning task. (3) An ACT-R model of TTB is developed, which uses as input the memory parameters estimated in the learning task. (4)

attribute and company are available in the model's buffers, the model attempts to recall the attribute value and responds by pressing a key on the keyboard. We computed the median duration of the nonretrieval processes over 100 runs $^{7}$ of that model to estimate the nonretrieval time. This was necessary because ACT-R assumes a certain variability in the operation times of its various cognitive components, such as visual attention and motor action. As a result, ACT-R makes predictions about the distributional characteristics of RTs. Our model estimated a mean

\footnotetext{
${ }^{7}$ We ran each model 100 times, which strikes a good balance between number of data points generated and the required simulation time.
}
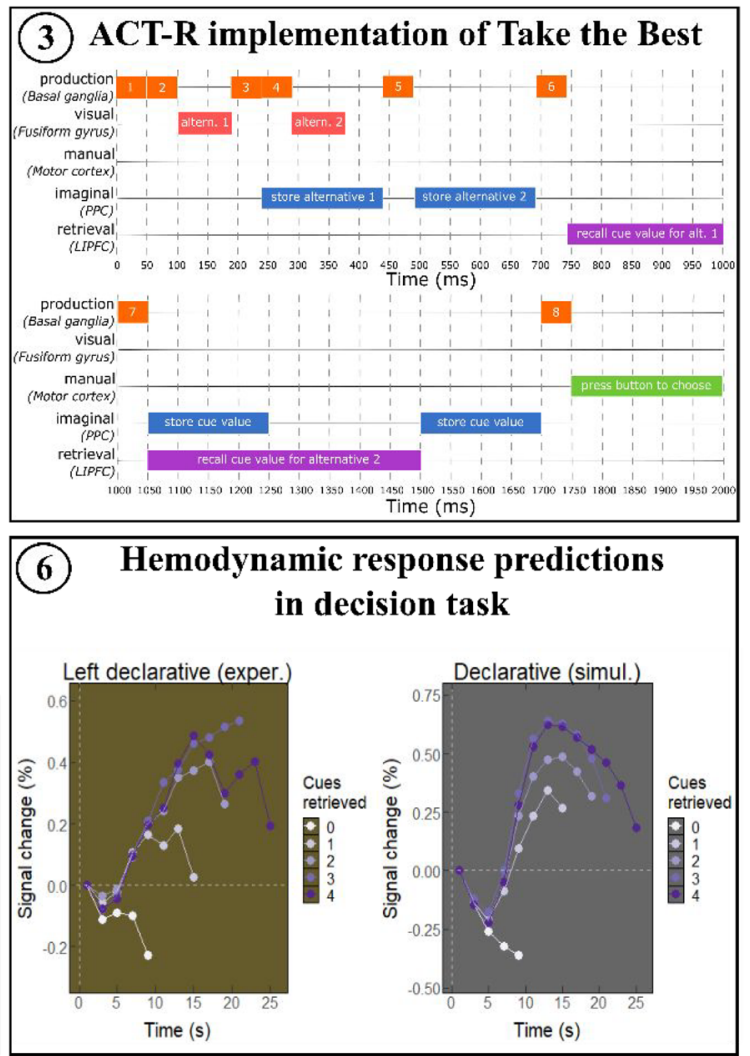

Distributional RT predictions are generated using this model and are compared with the experimental RTs. (5) Module activity is mapped onto hemodynamic response (HR). (6) The predicted HR is compared to the experimental fMRI data

perceptual-motor time of $780 \mathrm{~ms}$ for the first attribute of a company, and $495 \mathrm{~ms}$ for the remaining three attributes. ${ }^{8}$

After subtracting the average $t_{\text {nonretrieval }}$ from the overall RT, we removed outliers from the resulting $t_{\text {retrieval }}$ : Specifically, first we removed outliers on the left side of the distribution by eliminating all negative values; this amounted to $4.3 \%$ of all observations. Second, we removed outliers on the right side of the distribution by removing the $2.5 \%$ most extreme values (see Ratcliff, 1993, for general recommendations about outlier

\footnotetext{
${ }^{8}$ In the learning task of the experiment, attributes were grouped per company. Thus, a participant only needed to encode the company name for the first attribute.
} 


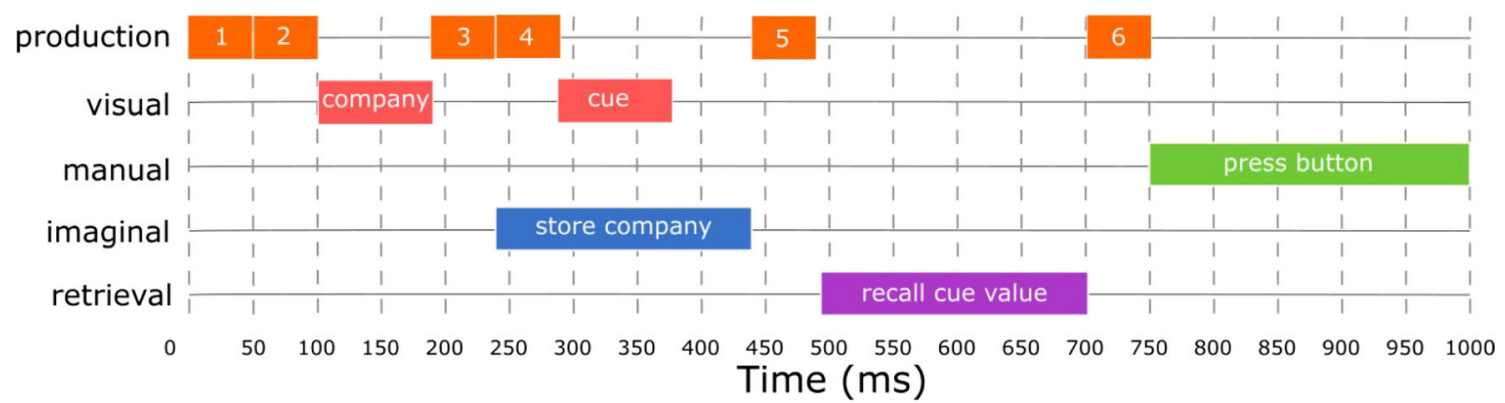

Fig. 5 Schematic process trace of ACT-R model of recall. The $y$-axis denotes various ACT-R modules. This model operates through six production steps. Productions 1 and 2 request that the visual module attend to and encode the company name, whereas Productions 3 and 4 direct the visual module to attend and encode the attribute. Production 5 tells the declarative module to recall the value for that attribute, and finally, Production 6 makes the appropriate response by requesting a keypress from the manual module. For the sake of illustration, the model trace schematically depicts somewhat arbitrary recall times and not those that a participant would likely need removal). The 97.5th percentile of the distribution of all participants' RTs was at $6.2 \mathrm{~s}$.

To account for memory retrieval being inherently noisy, ACT$\mathrm{R}$ models the noise on activation with a logistic distribution (see Eqs. 1 and 5). We assume that all 64 attribute value chunks stored in a participant's memory are characterized by the same parameter values (logistic distributions with equal means and scales; Fig. 4, Step 2). This assumption is plausible for three reasons. First, all attributes have approximately the same learning history (i.e., they were presented similarly often and similarly long ago) and hence the same base-level activation. Second, all attribute values receive the same amount of spreading activation, because every attribute is related to 16 attribute values (an associative fan of 16), and each company to four attribute values (a fan of 4). Third, by definition there is a single activation noise parameter per participant. Starting from Eq. 1, this assumption means that all chunks $i$ for a participant have an activation

$A_{i} \sim \operatorname{Logistic}\left(\mu_{A}, s\right)$.

In essence, three parameters have to be estimated per participant: the two parameters of the logistic distribution (mean activation $\mu_{A}$ and activation noise $s$ ), as well as a retrieval threshold $\tau$ (see Eqs. 6 and 8).

Figure 6 shows, for three representative participants, the resulting fits of a logistic distribution to the 64 samples from the activation distribution from the last round of learning (corresponding to the 64 attribute values). These 64 data points are RT distributions transformed using Eqs. 12 and 13. The parameters of such a theoretical cumulative distribution function provide an estimate of the mean activation and activation noise for all 64 attribute value chunks of a participant. To estimate each participant's retrieval threshold, we set it equal to the activation of the least active attribute value (see also Fig. 6).

\section{RT predictions}

The memory parameters, estimated individually for each participant, are used to derive predictions for the decision task:
Activation and other components feed into the TTB model described above (Fig. 4, Step 3). ${ }^{9}$ When generating predictions, all other parameters were set to their ACT-R default values. To mimic the exact experimental conditions, we added the timing details of an ITI of 2, 4, or $6 \mathrm{~s}$, a fixation cross presented for $2 \mathrm{~s}$ at the beginning of each trial, and presentation of a company pair until a response is made (see the description of the experimental procedure above) to the model presented in Fig. 3. When the fixation cross is drawn, the model looks at it. When an ITI is presented, the model does nothing. Finally, when the two companies are presented, the model executes TTB: It reads the company names sequentially, recalls attributes and attribute values, and responds with a keypress (see Fig. 14 in the Appendix for a trace of the complete final model, which also includes screen events and mental events for clarity).

As we mentioned in the ACT-R overview above, ACT-R assumes that cognitive processes are inherently noisy, implying that the same process can produce different patterns of data. To model this variation, ACT-R models are typically run multiple times in a computer simulation. In our case, the simulation of the complete decision task, consisting of 132 trials, was repeated 100 times for each participant. In so doing, in each simulation run and for each participant, the stimuli (i.e., pairs of company names) were presented in the same order in which the participants saw them. The resulting distributional RT predictions were compared against the empirical RTs (Fig. 4, Step 4). Figure 7 offers a snapshot of such a comparison, by plotting the median RTs and RT percentiles for three participants calculated by ACT-R, together with the empirical RTs for those participants (see the supplementary online materials for plots for all participants).

The trial-by-trial RT predictions of Participant 1 are summarized as a function of the number of attributes that needed

\footnotetext{
${ }^{9}$ Given that the time elapsed between last round of the learning task and the beginning of the decision task is minimal, we assume negligible memory decay between those tasks.
} 

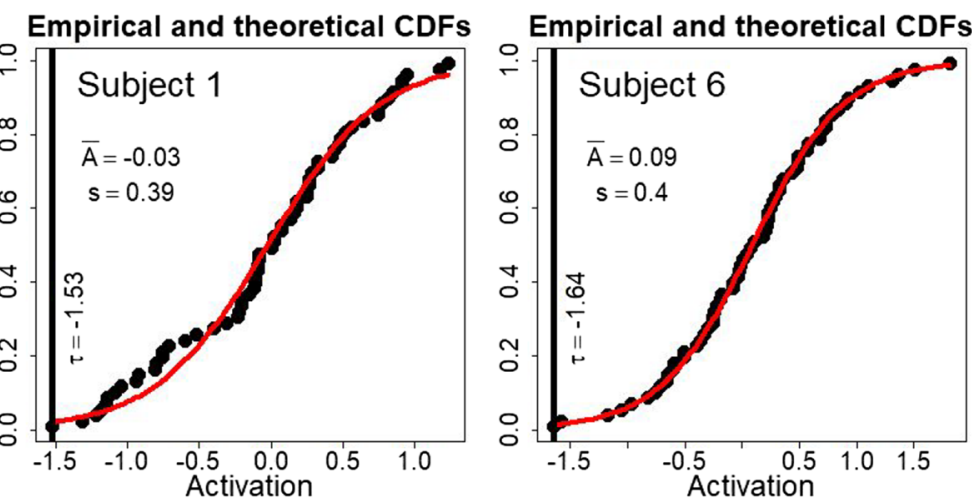

Empirical and theoretical CDFs

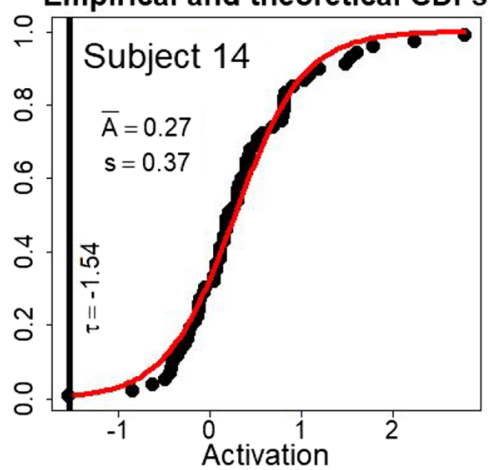

Fig. 6 Examples of fitting a logistic distribution to the learning data of three participants. Points show 64 empirically estimated activations from the last round of the learning task for each of the 64 attributes. The red

to be retrieved to make a decision and compared to the experimental data in Fig. $8^{10}$ (see the supplementary online materials for such plots for all participants). When the same company name is presented on both sides of the screen and, consequently, no attributes need to be retrieved, the model almost always responds within $1 \mathrm{~s}$ (Fig. 8a; median $_{\text {sim }}=918$ $\mathrm{ms})$, whereas this subject more frequently needed between 1 and $2 \mathrm{~s}\left(\right.$ median $\left._{\exp }=1,237 \mathrm{~ms}\right)$. Most participants (11 out of 17) also more frequently respond within $1 \mathrm{~s}$ on such trials. Moreover, as can be seen, for this participant, the more attributes need to be retrieved (one attribute, Fig. $8 \mathrm{~b}: \operatorname{median}_{\mathrm{sim}}=$ $3,788 \mathrm{~ms}$, median $_{\exp }=3,816 \mathrm{~ms}$; two attributes, Fig. $8 \mathrm{c}$ : median $_{\text {sim }}=6,923 \mathrm{~ms}$, median $n_{\text {exp }}=7,886 \mathrm{~ms}$; three or four attributes, Fig. $8 \mathrm{~d}$ : median $_{\text {sim }}=11,026 \mathrm{~ms}$, median $_{\exp }=$ $14,854 \mathrm{~ms}$ ), the more likely it is that his or her RTs will deviate from the model predictions. This trend can be seen among most subjects, with some subjects' data aligning better with our predictions, and some less well. We suspect that three factors contribute to this trend. First, the sample sizes are smaller when more attributes need to be retrieved, and thus, each sample is more variable. Second, RT variability also increases with increasing RTs. Finally, the probability of not precisely following the prescribed strategy (e.g., by getting distracted or wrongly remembering an attribute value) increases, the longer the execution of that strategy. Interestingly, some subjects exhibit very fast RTs (in some cases within $2 \mathrm{~s}$ ) even on trials that require three or four attributes to be considered, which supports our last hypothesis. When developing ACT-R models, detecting such deviations by plotting predictions and data in different ways is important. This aids in gauging the overall performance of a model and can uncover where further model refinements are warranted.

\footnotetext{
$\overline{10}$ There are few items, on which three attributes (16 items) or four attributes (eight items) need to be retrieved. As a consequence, we have grouped those items together to have a better representation of their distribution. Note that there are also only eight items, on which no attributes need to be retrieved, but these exhibit a very small variability in RT and are thus not grouped with other items.
}

lines show the fitted logistic curve, whereas the black lines designate the value of the retrieval threshold

To further illustrate this point and examine interparticipant variability, one can also ask how well our model performs across all participants. In Fig. 9 we compare the extents to which the empirically observed RTs deviate from the median predicted RTs (in terms of their mean absolute deviation, MAD), relative to how much the model deviates, on average, from the median predicted RTs on individual runs. How much the model deviates on each individual run from its median RT predictions provides us with an estimate of model variability. If a participant shows a smaller deviation from the median model predictions than individual model runs, our model is overestimating the variability in RTs. On the other hand, if a participant shows a larger MAD, our model is either underestimating a participant's RT variability or systematically deviating from the participant's RT. Figure 9 demonstrates that participants and model exhibit similar variabilities, although the empirical RTs typically depart more from the median predictions than do the individual runs of the simulation. Moreover, as we noted above, as a participant needs to retrieve more attributes (no attribute, Fig. 9a; one attribute, Fig. 9b; two attributes, Fig. 9c; three or four attributes, Fig. 9d), his/her RTs depart more strongly from the predictions.

\section{Neural activation predictions}

In the overview of ACT-R, we explained how HR predictions are derived in ACT-R. Figure 10 illustrates, in practice, how the HR resulting from a module's activity emerges (Eq. 11). In this figure, the activity of two modules (the declarative and visual modules), as described by their demand functions (light color), is transformed into predicted BOLD responses (dark color) for the first $50 \mathrm{~s}$ of a model run for a participant. Following the same procedure as for these $50 \mathrm{~s}$, we derived BOLD response predictions for each of the 100 model runs of each participant for the entire decision task (Fig. 4, Step 5). Thus, we were 

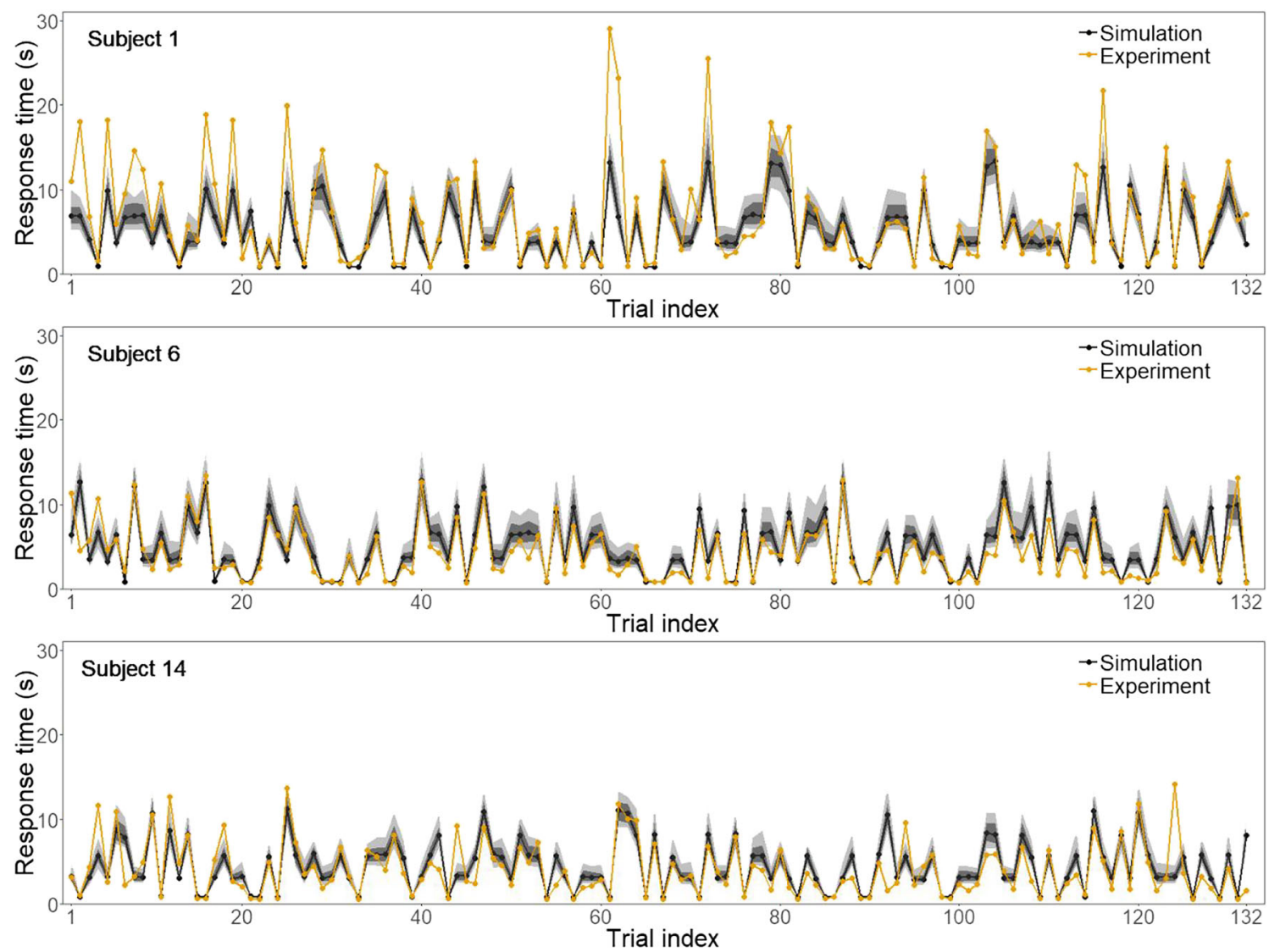

Fig. 7 Distributional RT predictions for three participants (over 100 runs) of the ACT-R model of TTB, and observed RTs of the corresponding participants in Khader et al. (2011, Exp. 1). The trial index is shown on the $x$-axis. That index can be thought of as a timeline, with the first trial corresponding to the first and the last trial to the last comparison of two

effectively able to specify the expected pattern of brain activation related to each of ACT-R's modules, given the sequence of cognitive steps assumed by our model.

When engaging in such ACT-R modeling, the details matter: For instance, we modeled the repeated presentation of fixation crosses and ITIs in the experimental procedure (see Fig. 14 in the Appendix for a process trace of the complete final model). This is important for accurately generating BOLD predictions, because the repeated presence of fixation crosses and ITIs shapes the time course of the HR. As can be seen in Fig. 2, the HRF needs more than 20 s to settle back to its baseline level. Given that the fixation cross duration is $2 \mathrm{~s}$ and that the ITI is at most $6 \mathrm{~s}$, there will always be some residual HR from the previous trial in the current trial (see the dashed lines in Fig. 10, which represent the time points at which a new trial begins).

Following the ROI procedure, these HR predictions, associated with different cognitive processes specified in the model, were compared to the observed brain activity. BOLD signals were extracted from module-specific areas based on the center coordinates and ROI sizes provided in J. R. Anderson companies in the decision task. The black lines represent the median predicted RTs across trials; the dark gray strips are the regions between the 25th and 75th RT percentiles; the light gray strips are the regions between the 10th and 90th RT percentiles. The observed RTs are presented in yellow. There were 132 paired comparisons of companies, in total

(2007). ${ }^{11}$ To mimic an fMRI scan, model predictions were averaged every $2 \mathrm{~s}$. Then, for both predictions and observations, the first scan of each trial for that participant (and that model run in the case of the predictions) served as baselineBOLD response was estimated relative to its magnitude. Finally, both the predicted and observed BOLD responses for all participants were grouped according to the number of attributes that had to be considered before a decision could be made, and averaged over bins of $2 \mathrm{~s}$. Figure 11 compares the predictions for the five modules of interest ${ }^{12}$ and observations from the corresponding regions in both the left and right hemispheres. The first column of Fig. 11 plots recordings from the left hemisphere, the second those from the right hemisphere, and the third column plots our model predictions.

\footnotetext{
${ }^{11}$ All coordinates are in Talairach space: declarative module: $x= \pm 43, y=23$, $z=24$; imaginal module: $x= \pm 23, y=-63, z=40$; procedural module: $x= \pm$ 14, $y=10, z=7$; manual module: $x= \pm 41, y=-20, z=50$; visual module: $x=$ $\pm 42, y=-61, z=-9$ (from J. R. Anderson, 2007, p. 189).

${ }^{12}$ ACT-R currently does not generate separate predictions for each hemisphere.
} 


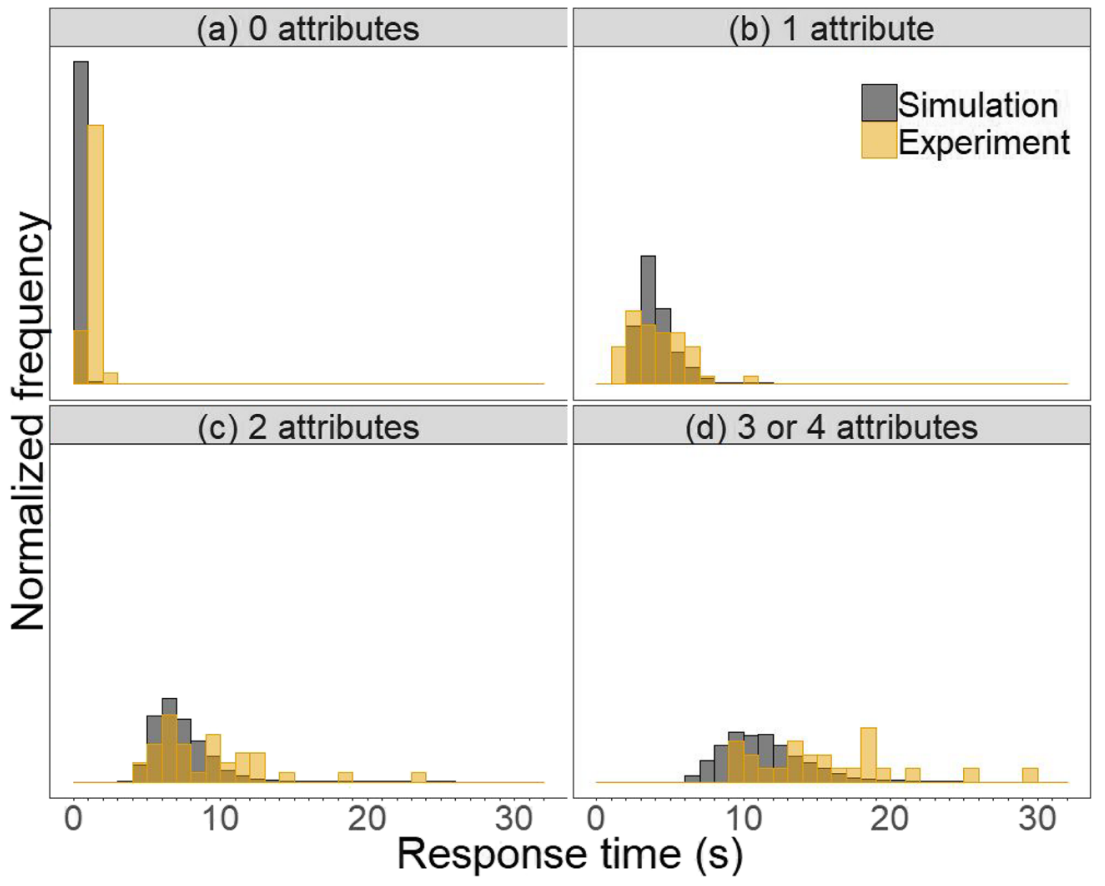

Fig. 8 Comparison of predicted and observed RT distributions in the decision task as a function of the number of attributes considered, for Participant 1 from Khader et al. (2011, Exp. 1). Each count corresponds to a trial for that participant

To quantify the degree of correspondence between the predicted and observed fMRI patterns, we used the Tucker congruence coefficient (TCC) ${ }^{13}$ and the coefficient of determination $R^{2}$ (similar to Borst et al., 2015). In addition, we used a weighted coefficient of determination $R_{\mathrm{w}}^{2}$ (computed as the square of the weighted correlation between predictions and observations), which weights each point by the number of observations that were averaged to produce that point, whereby the averaging took place over participants and trial times. Table 1 compares the predictions and observations on these three measures for the ten brain ROIs. The measures of correspondence between model and data are comparably good, in relation to the values others have found (e.g., the fit in Borst et al., 2015, which established new mappings of modules to brain regions, shows TCCs in the range between .86 and .96 and $R^{2} \mathrm{~S}$ in the range of .67 and .93). There were also two important deviations: First, the visual regions failed to match the observed increase in BOLD response amplitude with number of attributes considered. Second, the two motor regions correlated more weakly with the predicted BOLD response than did the remaining regions.

When developing ACT-R models, such discrepancies need to be explained and resolved so that they can, potentially, inform future model refinements. For instance, the weaker match between predictions and observations for the manual module was probably due to low motor activity: Motor activity (i.e., pressing

\footnotetext{
$\overline{13}$ TCC is used to assess the similarity of two quantities. Just like the correlation coefficient, it ranges between -1 and 1 . Unlike the correlation, TCC is based on the degree to which the quantities deviate from 0 , as opposed to from their means.
}

a key on the response device) was necessary only once, at the end of each trial. This, in turn, probably resulted in a low signal relative to the BOLD noise. We can probably do nothing to further improve the motor predictions of our model. However, predictions based on the visual module and BOLD data recorded in the corresponding brain region exhibited low correlation and negative TCC. The negative TCC in particular means that the observed signal tends to follow the opposite sign from our predictions: Whenever the observed signal is positive, our predictions tend to be negative and vice versa. A visual inspection of Fig. 11 further corroborates that our model fails to predict activity in the visual region. This might be due to a possible mismatch between our model and the sequence of cognitive processes that participants executed, calling for further refinement of our model to better match participants' visual activity.

\section{Model refinement}

There are often multiple ways of translating a model into a detailed architectural implementation. This holds also true when translating TTB's search, stopping, and decision rules into ACT-R. Our first ACT-R implementation of TTB relied on a set of assumptions about how people would execute this strategy. Yet, the ROI analysis outlined above demonstrated that some of those assumptions may not hold. This provides us with an opportunity to further refine our model. What other ways are there to translate TTB into ACT-R?

Currently, our model maintains the company names in a short-term store (i.e., the imaginal buffer). Yet a participant might avoid the burden of storing this information that is readily 


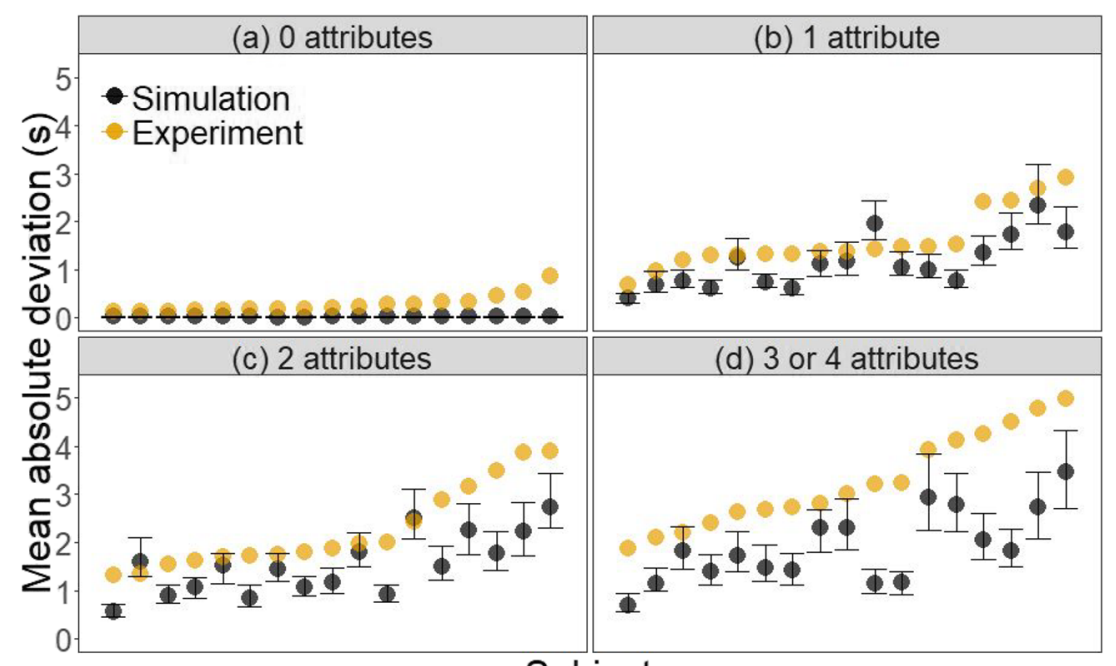

\section{Subject}

Fig. 9 Comparison of mean absolute deviations (MADs) of the individual model runs and of the experimental data from the median predicted RTs of the model, as a function of number of attributes retrieved before deciding. RT absolute deviations are averaged over participants and trial types (i.e., the number of attributes that need to be recalled). Error bars represent the minimum and maximum MADs from the 100 runs of the model

it to the imaginal buffer before comparing the attribute values. Finally, when we attempt to retrieve knowledge from long-term memory, we often shift our visual attention (i.e., we look away) and so then need to reallocate it back to its initial position (i.e., toward the screen), whereas for our model visual attention is always on the last object that it looked at. available on the computer screen by reading one or both company names off the screen upon the inspection of each attribute. Additionally, our model stores both attribute values in the imaginal buffer before comparing them. However, the second attribute value is immediately accessible after being recalled (i.e., in the retrieval buffer), and it might not be necessary to first move

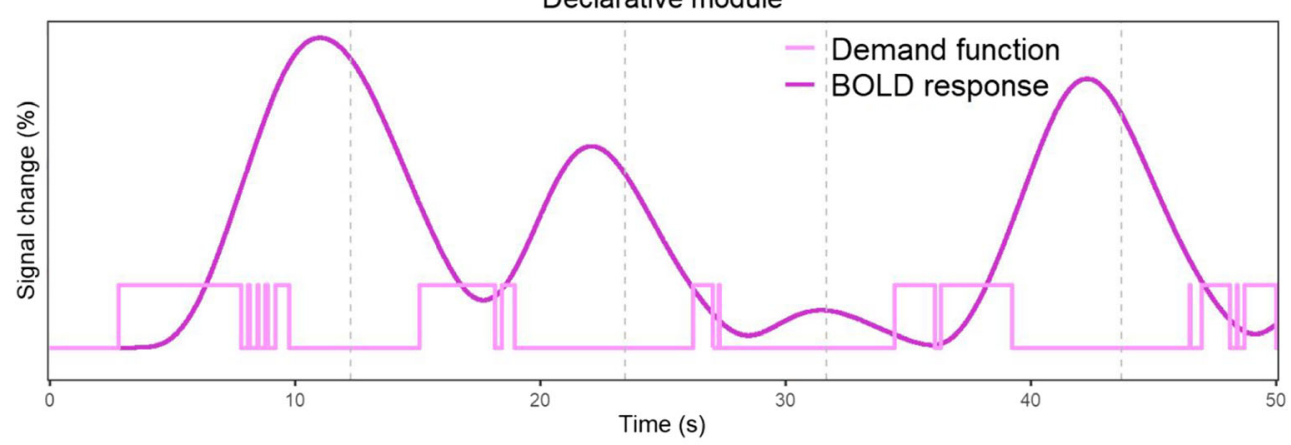

Visual module

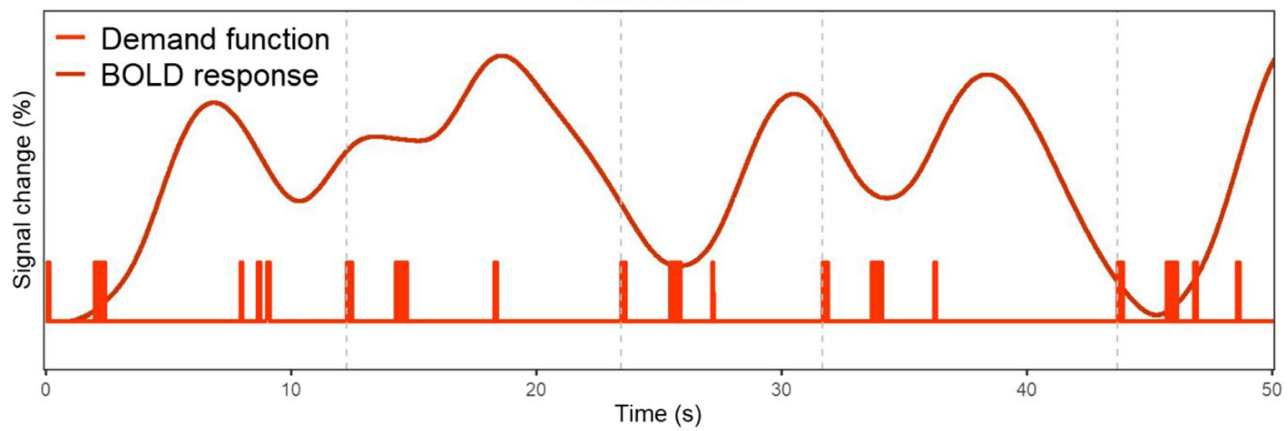

Fig. 10 Transformation of visual and retrieval module activity, as described by the demand function, to hemodynamic response (HR) for first $50 \mathrm{~s}$ of a run of an ACT-R implementation of TTB. The beginning of each trial is denoted with a dashed gray line. The HR often has not decayed to baseline before the beginning of the subsequent trial 

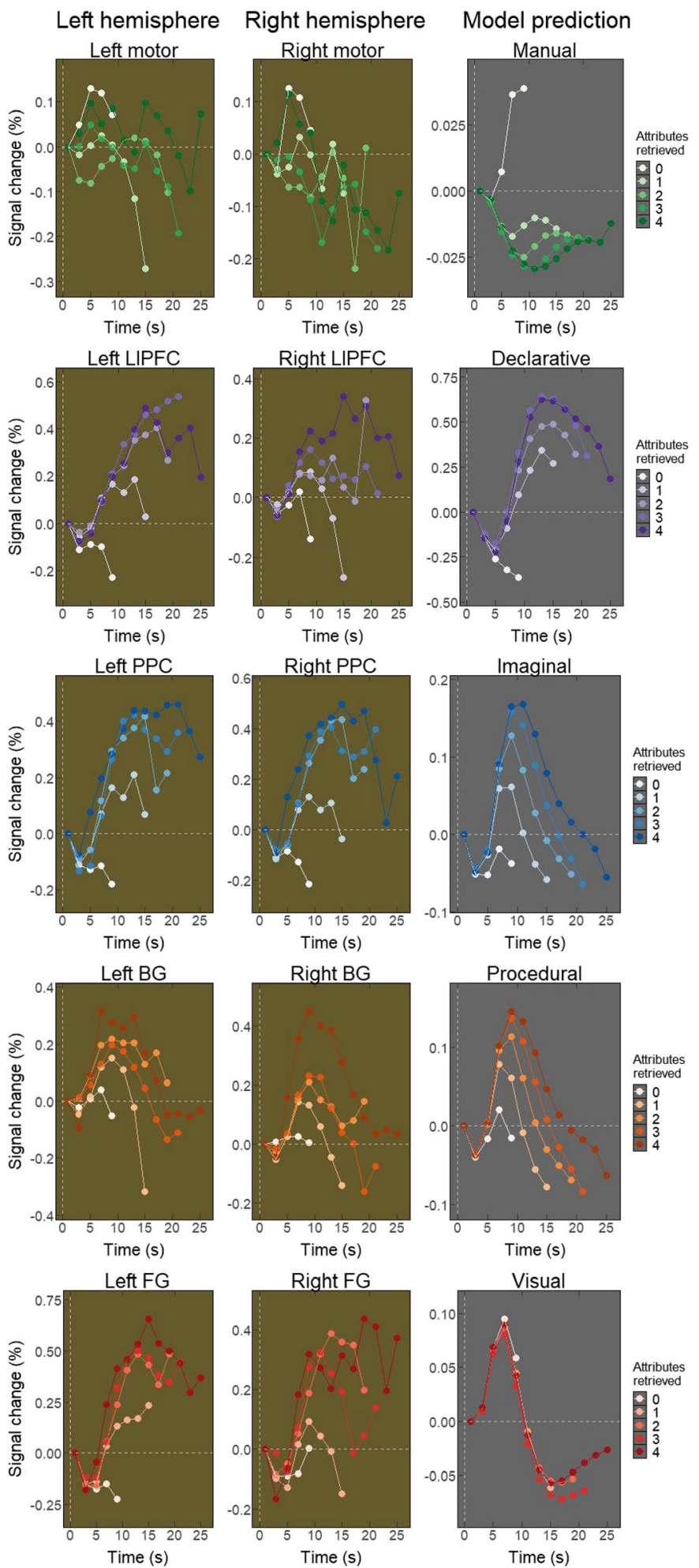

Fig. 11 Observed and predicted BOLD responses for ten brain regions, associated with the manual, declarative, imaginal, procedural, and visual modules in ACT-R. The $x$-axis represents the time point at which the BOLD signal is measured or predicted, whereas the $y$-axis represents the signal change relative to the first scan of that trial. The different degrees of brightness are associated with different numbers of attributes that need to be retrieved before TTB makes a decision. The BOLD

response is averaged over all participants and trials that require the respective number of attributes to be retrieved. Only points averaged from at least 20 observations are included in the plot, because the empirical data become very noisy with only a few observations. BG = basal ganglia, $\mathrm{FG}=$ fusiform gyrus, $\mathrm{LIPFC}=$ lateral inferior prefrontal cortex, $\mathrm{PPC}=$ posterior parietal cortex 
Table 1 Comparison of empirical and model brain activity for the brain regions corresponding to the five modules active in our TTB implementation

\begin{tabular}{lccc}
\hline Region & TCC & $R^{2}$ & $R_{\mathrm{w}}^{2}$ \\
\hline Left manual & .26 & .08 & .14 \\
Right manual & .62 & .21 & .33 \\
Left visual & -.51 & .48 & .29 \\
Right visual & -.33 & .20 & .18 \\
Left procedural & .80 & .61 & .73 \\
Right procedural & .84 & .73 & .80 \\
Left imaginal & .72 & .52 & .75 \\
Right imaginal & .75 & .58 & .76 \\
Left declarative & .94 & .86 & .87 \\
Right declarative & .66 & .30 & .40 \\
\hline
\end{tabular}

TCC = Tucker's congruence coefficient, $R_{\mathrm{w}}{ }^{2}=$ weighted coefficient of determination. $R_{\mathrm{w}}{ }^{2}$ is computed by squaring the weighted correlation between the predictions and observations

We created seven additional models (Table 2) that varied according to these dimensions. $\mathrm{TTB}_{1}$ is the model that we have examined up to this point. $\mathrm{TTB}_{2}$ reflects the intuition that people may access an attribute's value directly after they recalled it instead of first moving it to a short-term store. Further reducing the amount of information maintained in short-term memory, $\mathrm{TTB}_{3}$ and $\mathrm{TTB}_{4}$ read company names off the computer screen instead of storing them in the imaginal buffer. $\mathrm{TTB}_{5}$ lies at the intersection between $\mathrm{TTB}_{1} / \mathrm{TTB}_{2}$ and $\mathrm{TTB}_{3} / \mathrm{TTB}_{4}$, because it only maintains one company name in the imaginal buffer. Similarly, $\mathrm{TTB}_{6}$ and $\mathrm{TTB}_{7}$ are combinations of $\mathrm{TTB}_{1} / \mathrm{TTB}_{2}$ and $\mathrm{TTB}_{3} / \mathrm{TTB}_{4}$, as they not only look at both company names upon the inspection of each attribute, but also store those company names in the imaginal buffer. Finally, $\mathrm{TTB}_{8}$ is the model that loses the attentional focus once a retrieval starts, so that attention needs to be reallocated after retrieval is completed.

We submitted these eight implementations of TTB to a competitive test against each other. Although all models generated comparable behavioral predictions (see sections S2 and S3 in the supplementary online materials), their neural predictions (see section S4 in the supplementary online materials for figures) pointed toward the most plausible among the models.
Table 3 reports goodness-of-fit measures for the neural predictions of our initial TTB implementation $\left(\mathrm{TTB}_{1}\right)$, the bestfitting implementation $\left(\mathrm{TTB}_{8}\right)$, and an implementation with an intermediate fit $\left(\mathrm{TTB}_{4}\right)$.

These results illustrate the merits of an ROI analysis: Even though the behavioral measures produced by the models were very similar, the unique module-to-brain mappings afforded neural data to measure the contribution of each cognitive process separately. Put differently, variations in cognitive activity that lead to a noticeable change in a brain region's activity can only be identified with the help of neural data. Identifying the degree of activity of each cognitive function then allows us to point at the cognitive processes that most likely generated observed BOLD signals. In our case that is $\mathrm{TTB}_{8}$, which assumes that object names are not stored in the imaginal buffer. Instead, they are read off the computer screen every time they are needed. This model also assumes that visual attention is lost upon each retrieval attempt, and then recovered once retrieval is completed.

At the same time, these results illustrate another important aspect of ACT-R modeling: Cumulative theory building (see Marewski \& Olsson, 2009). Specifically, the ACT-R architecture has been cumulatively refined, updated, and extended over the past decades, on the basis of thousands of data points from experimental research from all over the world (current version: ACT-R 7). If one develops isolated models (e.g., of decision making or other cognitive processes) in ACT-R, a similar cumulative processes of theory building can take place. Typically, the model code is publicly shared, allowing for models developed on one data set (or a series of data sets) to be re-used and tested by researchers from other labs on other data sets. The next step of what can be thought of an iterative research process of model development and continuous testing would be to submit our TTB implementations to tests on new data sets. Conducting such tests would be particularly important for $\mathrm{TTB}_{2-8}$, because those seven implementations emerged after the fact, as a result of a refinement of $\mathrm{TTB}_{1}$. Hence, in a strict sense, Table 3 reports actual model predictions from foresight only for $\mathrm{TTB}_{1}$, but not for the other implementations, whose model structures we adjusted after the fact (although we did not reestimate the model parameters). The next competitive test of those models should therefore be conducted on new data.

Table 2 Summary descriptions of the eight implementations of TTB

\begin{tabular}{|c|c|c|c|c|c|c|c|c|}
\hline Assumed processing steps & $\mathrm{TTB}_{1}$ & $\mathrm{TTB}_{2}$ & $\mathrm{TTB}_{3}$ & $\mathrm{TTB}_{4}$ & $\mathrm{TTB}_{5}$ & $\mathrm{TTB}_{6}$ & $\mathrm{TTB}_{7}$ & $\mathrm{TTB}_{8}$ \\
\hline Store company names in imaginal buffer & $\mathbf{X}$ & $\mathbf{X}$ & & & & $\mathbf{X}$ & $\mathbf{X}$ & \\
\hline Look back and forth upon inspecting each attribute & & & $\mathbf{X}$ & $\mathbf{X}$ & & $\mathbf{X}$ & $\mathbf{X}$ & $\mathbf{X}$ \\
\hline Store second attribute value in imaginal buffer & $\mathbf{X}$ & & $\mathbf{X}$ & & & $\mathbf{X}$ & & $\mathbf{X}$ \\
\hline Store one company name in imaginal buffer & & & & & $\mathbf{X}$ & & & \\
\hline Look at company name again after attribute is retrieved & & & & & & & & $\mathbf{X}$ \\
\hline
\end{tabular}


Table 3 Goodness-of-fit measures of three of the eight TTB ACT-R implementations

\begin{tabular}{|c|c|c|c|c|c|c|c|c|c|}
\hline \multirow[t]{2}{*}{ Region } & \multicolumn{3}{|l|}{$\mathrm{TTB}_{1}$} & \multicolumn{3}{|c|}{$\mathrm{TTB}_{4}$} & \multicolumn{3}{|l|}{$\mathrm{TTB}_{8}$} \\
\hline & TCC & $R^{2}$ & $R_{\mathrm{w}}{ }^{2}$ & TCC & $R^{2}$ & $R_{\mathrm{w}}^{2}$ & TCC & $R^{2}$ & $R_{\mathrm{w}}{ }^{2}$ \\
\hline Left manual & .26 & .08 & .14 & .26 & .08 & .15 & .24 & .07 & .13 \\
\hline Right manual & .62 & .21 & .33 & .63 & .22 & .34 & .62 & .22 & .34 \\
\hline Left visual & -.51 & .48 & .29 & .08 & .02 & .01 & .75 & .45 & .70 \\
\hline Right visual & -.33 & .20 & .18 & .20 & .00 & .03 & .73 & .42 & .66 \\
\hline Left procedural & .80 & .61 & .73 & .77 & .56 & .68 & .68 & .38 & .55 \\
\hline Right procedural & .84 & .73 & .80 & .84 & .72 & .80 & .78 & .54 & .65 \\
\hline Left imaginal & .72 & .52 & .75 & .55 & .25 & .54 & .77 & .56 & .77 \\
\hline Right imaginal & .75 & .58 & .76 & .60 & .32 & .56 & .80 & .61 & .78 \\
\hline Left declarative & .94 & .86 & .87 & .94 & .85 & .88 & .95 & .87 & .89 \\
\hline Right declarative & .66 & .30 & .40 & .66 & .30 & .41 & .66 & .29 & .41 \\
\hline
\end{tabular}

Models differ primarily in how well they predict the signal in the fusiform gyrus. The winner of the model comparison is $\mathrm{TTB}_{8}$, which predicts well the brain activity related to the visual module. Neural predictions for the other five models can be found in section S4 in the supplementary online materials

\section{Summary}

We outlined how to develop an ACT-R model based on behavioral and neural data, using the TTB heuristic in memory-based decision making as a case in point. Importantly, the parameters of our model were constrained in a separate (i.e., learning) task, which as independent from and preceded the actual decision task (cf. Khader et al., 2011). The model was used to generate predictions about RTs and brain activation for a decision task, in which participants are instructed to use TTB to decide between two options, for which they had to retrieve decision-relevant information from memory. Overall, the RT predictions matched well both central tendencies and variability of individual participants' data. The brain activation predictions of the first model corresponded well to observed fMRI signals in regions associated with the manual, retrieval, imaginal, and procedural buffers, but failed to predict activity in the visual region. Pushed by this failure, we further refined our model by generating alternative hypotheses about the sequence of processing steps, implemented them as ACT-R models, and selected the model whose predictions best corresponded to the observed fMRI data.

\section{General discussion}

Cognitive activities, such as decision making, are the result of various interplaying cognitive resources. When trying to understand such activities, researchers are faced with the challenge of separating the contribution of each cognitive capacity. In this article, we provide a step-by-step methodological primer of how to separate the contribution of each cognitive capacity by implementing models in ACT-R, and how such models can be tested with both behavioral and neural data, and further refined. As an illustrative example, we focused on a commonly studied model of decision making, the TTB heuristic (Gigerenzer \& Goldstein, 1996). After estimating the free parameters of an ACT-R implementation of TTB using an independent learning task, we generated predictions about RTs and brain activation for a decision task, and tested those predictions on an fMRI dataset. Overall, both predicted RT distributions and temporal and spatial patterns of brain activity of our final model corresponded well to the observed data. Our results demonstrate that a properly specified and constrained model can predict both of these types of data, without any further adjustment of its parameters. As expected, if the components of that model are refined in an iterative process, the resulting models' fit can further increase. More broadly, our results illustrate how decision making research can be grounded in more general cognitive theories (see also Dimov, 2018; Dimov \& Link, 2017; Dimov, Marewski, \& Schooler, 2013, 2017; Dougherty et al., 1999; Fechner et al., 2016; Fechner, Pachur, \& Schooler, 2019; Fechner, Schooler, \& Pachur, 2018; Gonzalez, Lerch, \& Lebiere, 2003; Link, Marewski, \& Schooler, 2016; Marewski \& Mehlhorn, 2011; Marewski \& Schooler, 2011; Schooler \& Hertwig, 2005; Thomas, Dougherty, Sprenger, \& Harbison, 2008). In fact, this approach might become a trend that, so we and others think, has the potential to ultimately revolutionize the field - once entry-level barriers to complex architectural modeling tools such as ACT-R break away.

In what follows, we (a) compare how our models would do in predicting participants' RT data if we used a common set of parameters for all participants, (b) describe potential sources of individual differences, (c) explicitly outline the four modeling principles that we followed in our model developing efforts, and (d) refer readers to material relevant to ACT-R.

\section{Individual versus group parameters}

Two key features of our approach are, first, that we generate predictions (in our case, in the decision task) by estimating model parameters (i.e., mean activation, activation noise and retrieval threshold) on a different task from the task of interest (a learning task) and, second, that we do so separately for each participant. Constraining parameters in different tasks lends credence to architectural models (A. Newell, 1973b) and, more generally, reducing the number of free parameters is a widely accepted good practice in psychology. Thus, we call for fitting free parameters on tasks separate from the main task whenever possible. Yet, how important is the estimation of individual parameter values for participants?

At least since Estes (1956) it has been known that artifacts in parameter estimation can emerge if model parameters are fitted to group data. For example, only when fitting individual data could Estes and Maddox (2005) recover reasonable parameter 
values. Others have argued that averaged data can change the underlying functional form - for example, from exponential at the individual level to a power function at the group level (R. B. Anderson \& Tweney, 1997; Heathcote, Brown, \& Mewhort, 2000; Myung, Kim, \& Pitt, 2000). In our case, we addressed this question by estimating common parameters from the learning data of all participants and generating new predictions with our model. To this end, we pooled the RTs of all participants from the last round of the learning task, removed outliers, and fitted the memory parameters to those.

Figure 12 demonstrates the group parameter fits and compares them to the individual parameter estimates. Not surprisingly, the common parameters fall in-between the individual parameters: Where the individual mean activations range between -0.55 and 1.02 , the common mean activation is 0.26 ; where the estimated retrieval noise ranges between 0.3 and 0.88 , the common retrieval noise is 0.52 .

It can be expected that for participants with individual parameter values close to the group values, it would not matter much which values we used, whereas for participants with parameter values farther from the group parameter values, it would matter more. Figure 13 shows how much participants' RTs deviated from the model predictions with the common versus the individual parameters. For more than half of the participants, it does not matter whether we fit the parameters individually or to all participants. For one participant (the rightmost on the graph), the common parameter values do better than the individual ones that we estimated. Finally, for five of the 17 participants (five of the six leftmost ones), using individual parameters improves the predictive power of our models. The predictions for those participants are roughly between 0.5 and $1.7 \mathrm{~s}$ more accurate with the individually estimated parameters.

Which procedure-individual or group parameter estimation - should be adopted when developing ACT-R models? In general, we recommend working with individualized parameter values, because people differ in terms of their ability to, for instance, remember information. People also differ in terms of the statistical structure of the real-world environments they have encountered. For instance, if our experimental data set had not entailed fictitious but realistic stimulus names, one might expect that people with different prior real-world exposure histories to company names would have different activation levels for the same company names (see the section on ACT-R's subsymbolic system). Note that computing individualized parameter values does not lead to the problem of overfitting if, as we have done, the resulting models are tested with fixed parameter values on a different task for each participant. Pooling data, in turn, can became an interesting option when the number of observations available for each individual participant is sparse and when one has reasons to assume that individual differences across participants are negligible. That said, when working with pooled data the modeler should be aware of the problem that the average parameter values used might not correspond to any individual and also of the artifacts that might arise from estimating parameters from group data.

\section{Individual strategy differences}

Although individual parameter values lead to predictions that are at least as good as those from a common set of parameters for all participants, even with individually estimated parameters, our model does not capture all the variance in participants' data. This might partially be due to the limited size of the learning data sample, which does not allow us to perfectly estimate the values of memory parameter. Other, human, factors such as accumulating fatigue or distraction might also play a role. Yet another possibility for the unaccounted variance might lie in inter-participant variability in strategy selection. In decision making, it is well known that participants vary widely in the strategies they adopt when facing a decision problem and results typically consist of tendencies of switching from one strategy to another as certain experimental factors are

(a) Empirical and theoretical CDFs

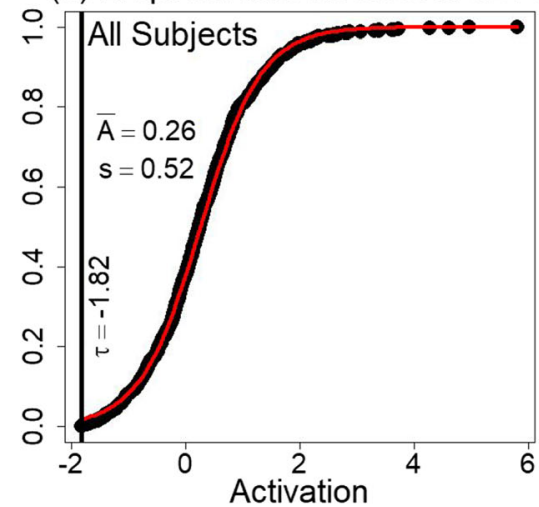

(b) Individual parameters

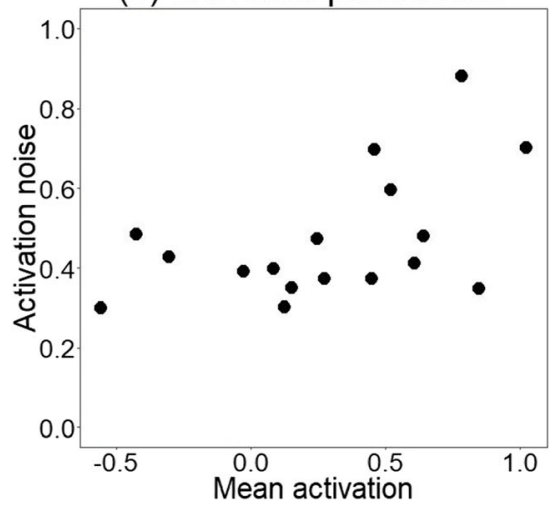

Fig. 12 a Activation fits of all observed response times in the learning task to generate common memory parameters for all participants. $\bar{A}$ denotes the estimated mean activation, $s$ the retrieval noise, and $\tau$ the retrieval threshold. $\mathbf{b}$ Individual parameter estimates for mean activation and activation noise 


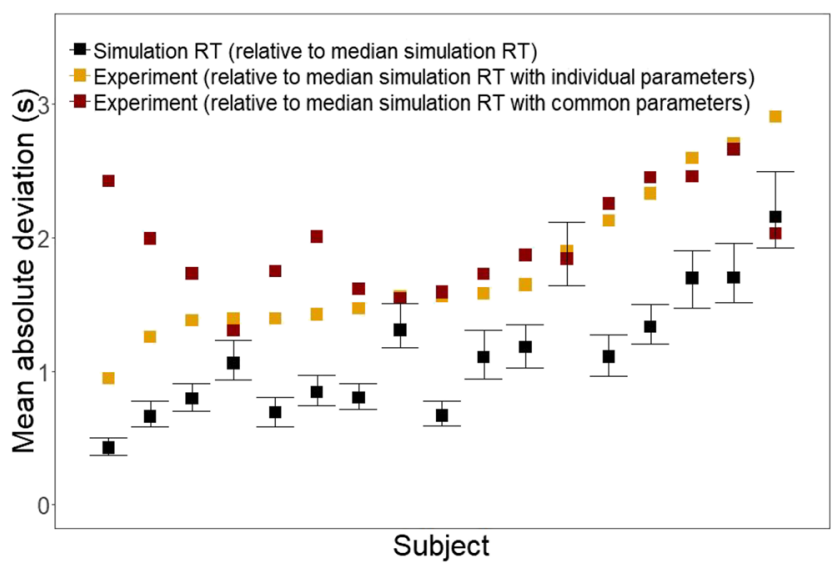

Fig. 13 Response time (RT) deviations from the median predicted RTs for model $\mathrm{TTB}_{1}$. In black is plotted the mean absolute deviations (MADs) of the individual runs of $\mathrm{TTB}_{1}$ (with memory parameters fitted separately for each participant) from the median RTs of that model. The MADs of each participant's RTs from the median predicted RTs of $\mathrm{TTB}_{1}$, with memory parameters fitted separately for each participant, are plotted in yellow. Red plots the MADs of each participant's RTs from the median predicted RTs of TTB $_{1}$ with memory parameters fitted to all participants' data from the learning task. The black dots estimate how much the simulation varies from run to run. Note that the participants' RTs (yellow) typically deviate more from the median model predictions than does any individual model run (see the black error bars, which delineate the region between the 10th and 90th percentiles of RTs on each model run). Moreover, for some participants, the participant RTs deviate more from a model whose memory parameters were fit to all subjects (in red) than from a model with individually estimated memory parameters (in yellow)

manipulated rather than a unanimous adoption of a single decision strategy (see, e.g., Bröder, 2012). Even in such a task as the one that we are modeling, in which participants are instructed to follow a particular strategy, each participant might still be executing TTB in an individually specific manner. In this case, different participants would best be described by different TTB implementations.

Another potential source of variability that we do not include in our analysis is the potential for strategy switching as the experiment progresses. Factors such as fatigue, exploration, or reinforcement (Rieskamp \& Otto, 2006) might slowly increase the likelihood that one implementation of TTB is executed rather than another. For example, a participant might start by storing all information in the short-term store (i.e., the imaginal module), similar to $\mathrm{TTB}_{1}$, and then discover that it is more efficient not to store information that is readily available to visual attention, as in $\mathrm{TTB}_{8}$. Exploring such individual differences is another potential pathway of model refinement.

\section{Principles for model development and testing in cognitive architectures}

Modeling frameworks as complex as ACT-R, which consists of multiple interplaying components and many adjustable parameters, are sometimes criticized for being capable of fitting everything (Pohl, 2011; Rieskamp \& Otto, 2006). Yet, such critique is misleading. ACT-R models make extremely precise multidimensional predictions (i.e., about different variables, including RT, BOLD signals, eye movements, etc.) that can be easily proved wrong in experiments. The analysis with our ACT-R implementations of TTB serve to illustrate, by means of a practical example, methods for creating strong test beds for architectural process models (see Fig. 4 for a roadmap of the steps that we followed). Specifically, we followed four modeling principles (Dimov \& Marewski, 2018).

First, although the ACT-R cognitive architecture has a certain number of free parameters, the calibration of those parameters is not arbitrary, nor is the careful modeler fully free to "choose" his or her own parameter values. A common standard in the ACT-R community is to use, wherever possible, ACT-R's default parameter values. If parameters need to be estimated, those parameters ought to be constrained on different data sets and tasks (see A. Newell, 1990; see also J. R. Anderson, 2007). In developing our model, we used the specified default parameter values if feasible and calibrated all models' free declarative parameters on the learning task, and then carried over those parameter values unchanged to the decision task (Fig. 4, Steps 1 and 2).

Second, ACT-R models ought to be tested in predicting new, unseen data, rather than in fitting existing data. Fitting refers to situations in which a model's free parameters are estimated from the human data by minimizing the difference between the humans and the model. In contrast, testing a model's predictions entails evaluating a model's ability to reproduce human data to which the model has not been calibrated - that is, out of sample - with fixed parameters (Marewski \& Olsson, 2009; Pitt et al., 2002; Roberts \& Pashler, 2000). After having developed our ACT-R implementation of TTB and calibrated its memory parameters on an independent behavioral task (see Fig. 4, Steps 1 and 2), we predicted, out of sample, participants' decisions, RTs, and neural patterns in the decision task (Fig. 4, Steps 3, 4, and 6).

Third, ACT-R models allow for making detailed quantitative predictions about distributions of human data, rather than merely predicting means, medians, or other point estimates (for a related approach, see Smith \& Ratcliff, 2004). Predicting the complexities of multiple distributions creates very strong tests for models, and often even merely trying to fit (rather than to predict) those distributions, poses a serious challenge. For example, Marewski and Mehlhorn (2011) specified 39 ACT-R models of decision making with none of them being able to fit and predict human RT distributions perfectly in two experiments. Yet, if only median RTs would have served as a criterion for model selection, several of those models would have been wrongly judged as being able to account equally well for human data. In our present analyses, we used our ACT-R implementation of TTB to predict distributional patterns (Fig. 4, Step 4).

Fourth, models are not evaluated in isolation, but according to their ability to make predictions, out of sample, relative to 
each other. In such model comparisons, models' predictions about dynamic distributional data, and not just means, should be contrasted. In this way, we might discover that no model accounts perfectly for both central tendencies and variabilities of various types of data, but, instead, we can establish the degree to which one model better predicts the data than another. The best model can then serve as a benchmark in future evaluations.

\section{ACT- $R$ resources}

In closing, we provide resources to get the reader started with ACT-R. First, the software package for the appropriate operating system can be downloaded from the ACT-R website (http://act-r. psy.cmu.edu/software/). In addition to the software, that website contains tutorials, a reference guide, and other documentation, which can aid the beginner modeler in immersing into the theory. Moreover, the website contains additional resources, such as a list of publications related to ACT-R and a list of researches working with this architecture. Publications cover a broad range of topics and usually come together with ACT-R models, which can be freely downloaded. For those willing to experiment with ACT-R without engaging with Common Lisp, versions in Java (jACT-R, http://jact-r.org/; Java ACT-R, http:// cog.cs.drexel.edu/act-r/) and Python (pyact, https://github.com/ jakdot/pyactr/; Python ACT-R, https://sites.google.com/site/ pythonactr/) exist, and the related ACTransfer theory and software (Taatgen, 2013) is written in Swift (to be downloaded from https://github.com/ntaatgen/ACTransfer). However, the most complete and up-to-date version of ACT-R is the one developed in Lisp, which since version 7.5 has included an interface that allows for interacting with the architecture through any programming language. Additionally, we refer those interested in modeling neural data with ACT-R to the excellent tutorial provided by Borst and Anderson (2017). Finally, we recommend that future (ACT-R) modelers seek out the literature on methods for model selection. A short overview (pertinent to ACT-R) is provided in Marewski and Olsson (2009).

Author note This research was supported by the Swiss National Science Foundation (grant number 100014_146702) awarded to J.N.M.

\section{Appendix}

Figure 14 is a description of a run of the final model, which extended the model described on Fig. 3 by adding an intertrial interval and a presentation cross. The presentation cross leads to additional visual activity when the model attends to the presented cross, whereas the inter-trial interval is a time of inactivity, during which the BOLD signal can decay. Thus, adding these two components to our model modifies its BOLD predictions. To simplify the understanding of the extended model, we have added screen events, which depict what is happening on the screen at this point in time, and mental events, which are higher level descriptions of what the model does at this point in time. For example, 2,000 ms after the trial starts, the two company names are presented on the screen (see the screen event). At this point, the model starts encoding the two company names off the screen (mental events "read company 1" and "read company 2") and, after determining that the names are different, starts executing TTB (mental event "names different; start TTB").

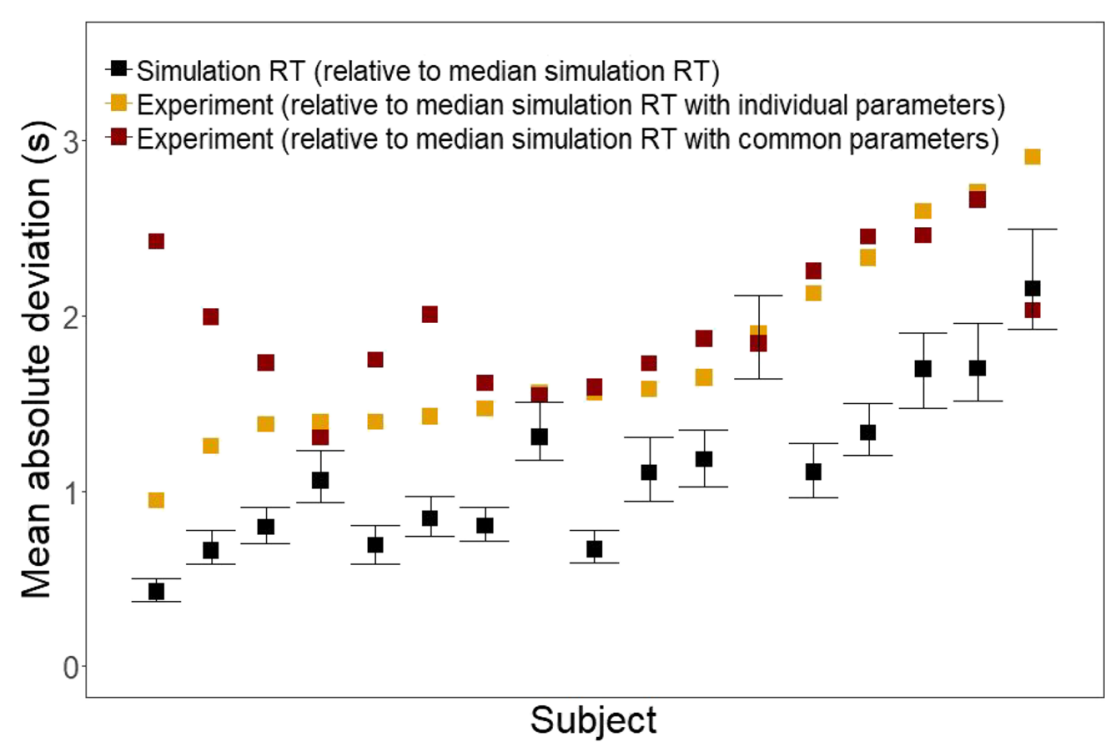

Fig. 14 Schematic process trace of an ACT-R implementation of TTB as applied to this experiment. The $y$-axis denotes various ACT-R modules and their associated brain regions. $L I P F C=$ lateral inferior prefrontal cortex. $P P C=$ posterior parietal cortex 


\section{References}

Anderson, J. R. (1990). The adaptive character of thought. Hillsdale, NJ: Erlbaum.

Anderson, J. R. (2007). How can the human mind occur in the physical universe? Oxford, UK: Oxford University Press.

Anderson, J. R., Bothell, D., Lebiere, C., \& Matessa, M. (1998). An integrated theory of list memory. Journal of Memory and Language, 38, 341-380. https://doi.org/10.1006/jmla.1997.2553

Anderson, J. R., Carter, C. S., Fincham, J. M., Qin, Y., Ravizza, S. M., \& Rosenberg-Lee, M. (2008). Using fMRI to test models of complex cognition. Cognitive Science, 32, 1323-1348. https://oi.org/10. 1080/03640210802451588

Anderson, J. R., Fincham, J. M., Qin, Y., \& Stocco, A. (2008). A central circuit of the mind. Trends in Cognitive Sciences, 12, 136-143. https://doi.org/10.1016/j.tics.2008.01.006

Anderson, J. R., \& Schooler, L. J. (1991). Reflections of the environment in memory. Psychological Science, 2, 396-408. https://doi.org/10. 1111/j.1467-9280.1991.tb00174.x

Anderson, R. B., \& Tweney, R. D. (1997). Artifactual power curves in forgetting. Memory \& Cognition, 25, 724-730. https://doi.org/10. 3758/BF03211315

Bergert, F. B., \& Nosofsky, R. M. (2007). A response-time approach to comparing generalized rational and take-the-best models of decision making. Journal of Experimental Psychology: Learning, Memory, and Cognition, 33, 107-129. https://doi.org/10.1037/0278-7393.33. 1.107

Bobadilla-Suarez, S., \& Love, B. C. (2018). Fast or frugal, but not both: Decision heuristics under time pressure. Journal of Experimental Psychology: Learning, Memory, and Cognition, 44, 24-33. https:// doi.org/10.1037/xlm0000419

Borst, J. P., \& Anderson, J. R. (2015). Using the ACT-R cognitive architecture in combination with fMRI data. In B. Forstmann \& E.-J. Wagenmakers (Eds.), An introduction to model-based cognitive neuroscience (pp. 339-352). New York, NY: Springer.

Borst, J. P., \& Anderson, J. R. (2017). A step-by-step tutorial on using the cognitive architecture ACT-R in combination with fMRI data. Journal of Mathematical Psychology, 76, 94-103. https://doi.org/ 10.1016/j.jmp.2016.05.005

Borst, J. P., Nijboer, M., Taatgen, N. A., van Rijn, H., \& Anderson, J. R. (2015). Using data-driven model-brain mappings to constrain formal models of cognition. PLoS ONE, 10, e0119673. https://doi.org/ 10.1371/journal.pone.0119673

Borst, J. P., Taatgen, N. A., Stocco, A., \& van Rijn, H. (2010). The neural correlates of problem states: Testing fMRI predictions of a computational model of multitasking. PLoS ONE, 5, e12966. https://doi. org/10.1371/journal.pone.0012966

Boynton, G. M., Engel, S. A., Glover, G. H., \& Heeger, D. J. (1996). Linear systems analysis of functional magnetic resonance imaging in human V1. Journal of Neuroscience, 16, 4207-4221. https://doi. org/10.1523/JNEUROSCI.16-13-04207.1996

Bröder, A. (2000). Assessing the empirical validity of the "Take-the-best" heuristic as a model of human probabilistic inference. Journal of Experimental Psychology: Learning, Memory, and Cognition, 26, 1332-1346. https://doi.org/10.1037/0278-7393.26.5.1332

Bröder, A. (2003). Decision making with the "adaptive toolbox": Influence of environmental structure, intelligence, and working memory load. Journal of Experimental Psychology: Learning, Memory, and Cognition, 29, 611-625. https://doi.org/10.1037/ 0278-7393.29.4.611

Bröder, A. (2012). The quest for take the best-Insights and outlooks from experimental research. In P. Todd, G. Gigerenzer, \& the ABC Research Group, Ecological rationality: Intelligence in the world (pp. 216-240), New York: Oxford University Press.
Bröder, A., \& Gaissmaier, W. (2007). Sequential processing of cues in memory-based multiattribute decisions. Psychonomic Bulletin \& Review, 14, 895-900. https://doi.org/10.3758/BF03194118

Bröder, A., \& Schiffer, S. (2003). Take The Best versus simultaneous feature matching: Probabilistic inferences from memory and effects of representation format. Journal of Experimental Psychology: General, 132, 277-293. https://doi.org/10.1037/0096-3445.132.2. 277

Byrne, M. D., \& Anderson, J. R. (2001). Serial modules in parallel: The psychological refractory period and perfect time-sharing. Psychological Review, 108, 847-869. https://doi.org/10.1037/ 0033-295X.108.4.847

Byrne, M. D., \& Kirlik, A. (2005). Using computational cognitive modeling to diagnose possible sources of aviation error. The International Journal of Aviation Psychology, 15, 135-155. https://doi.org/10. 1207/s15327108ijap1502 2

Chase, V. M., Hertwig, R., \& Gigerenzer, G. (1998). Visions of rationality. Trends in Cognitive Sciences, 2, 206-214. https://doi.org/10. 1016/j.tics.2004.11.005

Dimov, C. M. (2018). How to implement HyGene into ACT-R. Journal of Cognitive Psychology, 30, 163-176. https://doi.org/10.1080/ 20445911.2017.1394863

Dimov, C. M., \& Link, D. (2017). Do people order cues by retrieval fluency when making probabilistic inferences? Journal of Behavioral Decision Making, 4, 843-854. https://doi.org/10.1002/ bdm.

Dimov, C. M., \& Marewski, J. N. (2018). Cognitive Architectures as Scaffolding for Risky Choice Models. In M. Raue, E. Lermer, B. Streicher (Eds.), Psychological Perspectives on risk and risk analysis (pp. 201-216). Springer, Cham.

Dimov, C. M., Marewski, J. N., \& Schooler, L. J. (2013). Constraining ACT-R models of decision strategies: An experimental paradigm. In M. Knauff, M. Pauen, N. Sebanz, \& I. Wachsmuth (Eds.), Proceedings of the 35th Annual Conference of the Cognitive Science Society (pp. 2201-2206). Austin, TX: Cognitive Science Society.

Dimov, C. M., Marewski, J. N., \& Schooler, L. J. (2017). Architectural process models of decision making: Toward a model database. In G. Gunzelmann, A. Howes, T. Tenbrink, \& E. J. Davelaar (Eds.), Proceedings of the 39th Annual Conference of the Cognitive Science Society (pp. 1931-1936). Austin, TX: Cognitive Science Society.

Dougherty, M. R. P., Gettys, C. F., \& Ogden, E. E. (1999). Minerva-DM: A memory processes model for judgments of likelihood. Psychological Review, 106, 180-209. https://doi.org/10.1037/ 0033-295X.106.1.180

Duchowski, A. T. (2002). A breadth-first survey of eye-tracking applications. Behavior Research Methods, Instruments, \& Computers, 34, 455-470. https://doi.org/10.3758/BF03195475

Edwards, W. (1954). The theory of decision making. Psychological Bulletin, 51, 380-417. https://doi.org/10.1037/h0053870

Eliasmith, C. (2013). How to build a brain: A neural architecture for biological cognition. Oxford University Press, Oxford.

Ericsson, K. A., \& Simon, H. A. (1980). Verbal reports as data. Psychological Review, 87, 215-251. https://doi.org/10.1037/0033295X.87.3.215

Estes, W. K. (1956). The problem of inference from curves based on group data. Psychological Bulletin, 53, 134-140. https://doi.org/ $10.1037 / \mathrm{h} 0045156$

Estes, W. K., \& Maddox, W. T. (2005). Risks of drawing inferences about cognitive processes from model fits to individual versus average performance. Psychonomic Bulletin \& Review, 12, 403-408. https://doi.org/10.3758/BF03193784

Fechner, H. B., Pachur, T., \& Schooler, L. J. (2019). How does aging impact decision making? The contribution of cognitive decline and strategic compensation revealed in a cognitive architecture. Journal 
of Experimental Psychology: Learning, Memory, and Cognition. Advance online publication. https://doi.org/10.1037/xlm0000661

Fechner, H. B., Pachur, T., Schooler, L. J., Mehlhorn, K., Battal, C., Volz, K. G., \& Borst, J. P. (2016). Strategies for memory-based decision making: Modeling behavioral and neural signatures within a cognitive architecture. Cognition, 157, 77-99. https://doi.org/10.1016/j. cognition.2016.08.011

Fechner, H. B., Schooler, L. J., \& Pachur, T. (2018). Cognitive costs of decision-making strategies: A resource demand decomposition analysis with a cognitive architecture. Cognition, 170, 102-122. https:// doi.org/10.1016/j.cognition.2017.09.003

Friston, K. J., Fletcher, P., Josephs, O., Holmes, A. P., Rugg, M. D., \& Turner, R. (1998). Event-related fMRI: Characterising differential responses. NeuroImage, 7, 30-40. https://doi.org/10.1006/nimg. 1997.0306

Gigerenzer, G., \& Gaissmaier, W. (2011). Heuristic decision making. Annual Review of Psychology, 62, 451-482. https://doi.org/10. 1146/annurev-psych-120709-145346

Gigerenzer, G., \& Goldstein, D. G. (1996). Reasoning the fast and frugal way: Models of bounded rationality. Psychological Review, 104, 650-669. https://doi.org/10.1037/0033-295X.103.4.650

Glöckner, A. (2009). Investigating intuitive and deliberate processes statistically: The multiple-measure maximum likelihood strategy classification method. Judgment and Decision Making, 4, 186-199.

Gluck, K. A. (2010). Cognitive architectures for human factors in aviation. In E. Salas \& D. Maurino (Eds.), Human factors in aviation (2nd ed., pp. 375-400). New York, NY: Elsevier.

Gonzalez, C., Lerch, J. F., \& Lebiere, C. (2003). Instance-based learning in dynamic decision making. Cognitive Science, 27, 591-635. https://doi.org/10.1016/S0364-0213(03)00031-4

Heathcote, A., Brown, S., \& Mewhort, D. J. K. (2000). The power law repealed: The case for an exponential law of practice. Psychonomic Bulletin \& Review, 7, 185-207. https://doi.org/10.3758/ BF03212979

Hertwig, R., Herzog, S. M., Schooler, L. J., \& Reimer, T. (2008). Fluency heuristic: A model of how the mind exploits a by-product of information retrieval. Journal of Experimental Psychology: Learning, Memory, and Cognition, 34, 1191-1206. https://doi.org/10.1037/ a0013025

Johnson, E. J., Schulte-Mecklenbeck, M., \& Willemsen, M. C. (2008). Process models deserve process data: Comment on Brandstätter, Gigerenzer, and Hertwig (2006). Psychological Review, 115, 263272. https://doi.org/10.1037/0033-295X.115.1.263

Juslin, P., Jones, S., Olsson, H., \& Winman, A. (2003). Cue abstraction and exemplar memory in categorization. Journal of Experimental Psychology: Learning, Memory, and Cognition, 29, 924-941. https://doi.org/10.1037/0278-7393.29.5.924

Khader, P. H., Pachur, T., \& Jost, K. (2013). Automatic activation of attribute knowledge in heuristic inference from memory. Psychonomic Bulletin and Review, 20 (2), 372-377, https://doi. org/10.3758/s13423-012-0334-7

Khader, P H., Pachur, T., Meier, S., Bien, S., Jost, K., \& Rösler, F. (2011). Memory-based decision making with heuristics involves increased activation of decision-relevant memory representations. Journal of Cognitive Neuroscience, 23, 3540-3554. https://doi.org/10.1162/ jocn_a 00059

Lewandowsky, S. (1993). The rewards and hazards of computer simulations. Psychological Science, 4, 236-243. https://doi.org/10.1111/j. 1467-9280.1993.tb00267.x

Link, D., Marewski, J. N, \& Schooler, L. J. (2016). An ecological model of memory and inferences. In A. Papafragou, D. Grodner, D. Mirman, \& J.C. Trueswell (Eds.), Proceedings of the 38th Annual Conference of the Cognitive Science Society (pp. 1883-1888). Austin, TX: Cognitive Science Society.
Marewski, J. N., \& Mehlhorn, K. (2011). Using the ACT-R architecture to specify 39 quantitative process models of decision making. Judgment and Decision Making, 6, 439-519.

Marewski, J. N., \& Olsson, H. (2009). Beyond the null ritual: Formal modeling of psychological processes. Zeitschrift für Psychologie/ Journal of Psychology, 217, 49-60. https://doi.org/10.1027/00443409.217.1.49

Marewski, J. N., \& Schooler, L. J. (2011). Cognitive niches: An ecological model of strategy selection. Psychological Review, 118, 393437. https://doi.org/10.1037/a0024143

Meyer, D. E., \& Kieras, D. E. (1997). A computational theory of executive cognitive processes and multiple-task performance: Part 2. Accounts of psychological refractory-period phenomena. Psychological Review, 104, 749-791. https://doi.org/10.1037/ 0033-295X.104.4.749

Myung, I. J., Kim, C., \& Pitt, M. A. (2000). Toward an explanation of the power law artifact: Insights from response surface analysis. Memory \& Cognition, 28, 832-840. https://doi.org/10.3758/BF03198418

Newell, A. (1973a). Production systems: Models of control structures. In W. G. Chase (Ed.), Visual information processing (pp. 463-526). New York, NY: Academic Press.

Newell, A. (1973b). You can't play 20 questions with nature and win: Projective comments on the papers of this symposium. In W. G. Chase (Ed.), Visual information processing (pp. 283-310). New York, NY: Academic Press.

Newell, A. (1990). Unified theories of cognition. Cambridge, MA: Harvard University Press.

Newell, B. R., \& Lee, M. D. (2011). The right tool for the job? Comparing an evidence accumulation and a naive strategy selection model of decision making. Journal of Behavioral Decision Making, 24, 456-481. https://doi.org/10.1002/bdm.703

Newell, B. R., Weston, N. J., \& Shanks, D. R. (2003). Empirical tests of a fast-and-frugal heuristic: Not everyone "takes-the-best." Organizational Behavior and Human Decision Processes, 91, 8296. https://doi.org/10.1016/S0749-5978(02)00525-3

Nosofsky, R. M., \& Bergert, F. B. (2007). Limitations of exemplar models of multi-attribute probabilistic inference. Journal of Experimental Psychology: Learning, Memory, and Cognition, 33, 999-1019. https://doi.org/10.1037/0278-7393.33.6.999

Pachur, T., \& Aebi-Forrer, E. (2013). Selection of decision strategies after conscious and unconscious thought. Journal of Behavioral Decision Making, 26, 477-488. https://doi.org/10.1002/bdm.1780

Pachur, T., \& Bröder, A. (2013). Judgment: A cognitive processing perspective. Wiley Interdisciplinary Reviews: Cognitive Science, 4, 665-681. https://doi.org/10.1002/wcs.1259

Pachur, T., Hertwig, R., Gigerenzer, G., \& Brandstätter, E. (2013). Testing process predictions of models of risky choice: A quantitative model comparison approach. Frontiers in Psychology, 4. https://doi.org/10. 3389/fpsyg.2013.00646

Pachur, T., \& Marinello, G. (2013). Expert intuitions: How to model the decision strategies of airport customs officers? Acta Psychologica, 144, 97-103. https://doi.org/10.1016/j.actpsy.2013.05.003

Payne, J. W., Bettman, J. R., \& Johnson, E. J. (1993). The adaptive decision maker. New York: Cambridge University Press.

Pitt, M. A., Myung, I. J., \& Zhang, S. (2002). Toward a method of selecting among computational models of cognition. Psychological Review, 109, 472-491. https://doi.org/10.1037/ 0033-295X.109.3.472

Pohl, R. F. (2011). On the use of recognition in inferential decision making: An overview of the debate. Judgment and Decision Making, 6, 423-438.

Rescorla, R. A., \& Wagner, A. R. (1972). A theory of Pavlovian conditioning: Variations in the effectiveness of reinforcement and nonreinforcement. In A. H. Black \& W. F. Prokasy (Eds.), Classical conditioning II: Current research and theory (pp. 6499). New York, NY: Appleton-Century-Crofts. 
Rieskamp, J., \& Otto, P. (2006). SSL: A theory of how people learn to select strategies. Journal of Experimental Psychology: General, 135, 207-236. https://doi.org/10.1037/0096-3445.135.2.207

Ritter, S., Anderson, J. R., Koedinger, K. R., \& Corbett, A. (2007). Cognitive tutor: Applied research in mathematics education. Psychonomic Bulletin \& Review, 14, 249-255. https://doi.org/10. 3758/BF03194060

Roberts, S., \& Pashler, H. (2000). How persuasive is a good fit? A comment on theory testing. Psychological Review, 107, 358-367. https://doi.org/10.1037/0033-295X.107.2.358

Salvucci, D. D., \& Taatgen, N. A. (2008). Threaded cognition: An integrated theory of concurrent multitasking. Psychological Review, 115, 101-130. https://doi.org/10.1037/0033-295X.115.1.101

Schooler, L. J., \& Anderson, J. R. (1997). The role of processes in the rational analysis of memory. Cognitive Psychology, 32, 219-250. https://doi.org/10.1006/cogp.1997.0652

Schooler, L. J., \& Hertwig, R. (2005). How forgetting aids heuristic inference. Psychological Review, 112, 610-628. https://doi.org/10. 1037/0033-295X.112.3.610

Smith, P. L., \& Ratcliff, R. (2004). Psychology and neurobiology of simple decisions. Trends in Neurosciences, 27, 161-168. https:// doi.org/10.1016/j.tins.2004.01.006

Stocco, A., Lebiere, C., \& Anderson, J. R. (2010). Conditional routing of information to the cortex: A model of the basal ganglia's role in cognitive coordination. Psychological Review, 117, 541-574. https://doi.org/10.1037/a0019077
Taatgen, N. A. (2013). The nature and transfer of cognitive skills. Psychological Review, 120, 439-471. https://doi.org/10.1037/ a0033138

Taatgen, N. A., Huss, D., Dickison, D., \& Anderson, J. R. (2008). The acquisition of robust and flexible cognitive skills. Journal of Experimental Psychology: General, 137, 548-565. https://doi.org/ 10.1037/0096-3445.137.3.548

Thomas, R. P., Dougherty, M. R., Sprenger, A. M., \& Harbison, J. (2008). Diagnostic hypothesis generation and human judgment. Psychological Review, 115, 155-185. https://doi.org/10.1037/ 0033-295X.115.1.155

Tversky, A. (1972). Elimination by aspects: A theory of choice. Psychological Review, 79, 281-299. https://doi.org/10.1037/ h0032955

Willemsen, M. C., \& Johnson, E. J. (2011). Visiting the decision factory: Observing cognition with MouselabWEB and other information acquisition methods. In M. Schulte-Mecklenbeck, A. Kühberger, \& R. Ranyard (Eds.), A handbook of process tracing methods for decision research: A critical review and user's guide (pp. 21-42). New York, NY: Taylor \& Francis.

Publisher's note Springer Nature remains neutral with regard to jurisdictional claims in published maps and institutional affiliations. 